\title{
Active Flow Control for Trailing Edge Flap SEPARATION
}

\author{
LaTunia Pack Melton, Mehti Koklu† Marlyn Andino \\ NASA Langley Research Center, Hampton, Virginia, 23681 \\ Eli Shellabarger \\ Iowa State University, Ames, IA, 50011
}

\begin{abstract}
Active flow control (AFC) in the form of sweeping jet (SWJ) excitation and discrete steady jet excitation is used to control the flow separation on an NACA 0015 semispan wing with a deflected, simple-hinged, trailing edge flap. This geometry has been the focus of several recent publications that investigated methods to improve the efficiency of sweeping jet actuators. In the current study, the interaction of the AFC excitation with the separated flowfields present at several flap deflection angles was examined. Previous studies with this model have been limited to a maximum flap deflection angle of $40^{\circ}$. The flap deflection range was extended to $60^{\circ}$ because systems studies have indicated that a high-lift system with simple-hinged flaps may require larger flap deflections than the Fowler flaps found on most high-lift systems. The results obtained at flap deflection angles of $20^{\circ}, 40^{\circ}$, and $60^{\circ}$ are presented and compared. Force and moment data, Particle Image Velocimetry (PIV) data, and steady and unsteady surface pressure data are used to describe the flowfield with and without AFC. With a flap deflection of $60^{\circ}$, increasing the SWJ actuator momentum at the flap shoulder increased lift due to an increase in circulation but did not completely eliminate the recirculation region above the flap surface. AFC using the discrete steady jet actuators of this study increased lift as well but required more mass flow than the SWJ actuators and had a detrimental effect on lift at the highest mass flow level tested. PIV results showed that the angle between the excitation and the flap surface was not optimal for attaching the separated shear layer.
\end{abstract}

\section{Nomenclature}

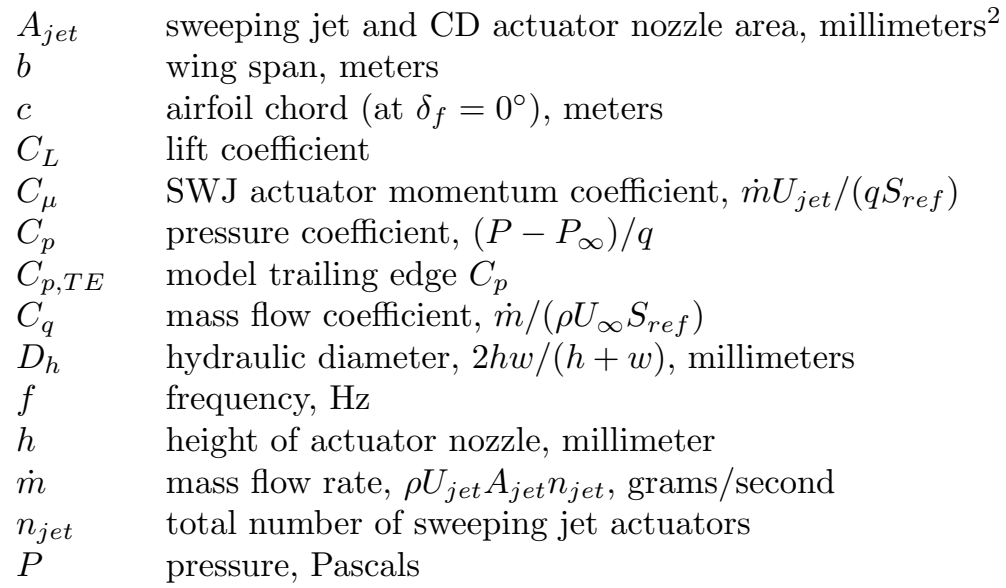

\footnotetext{
*Research Scientist, Flow Physics and Control Branch, Associate Fellow AIAA

${ }^{\dagger}$ Research Scientist, Flow Physics and Control Branch, Member AIAA

${ }^{\ddagger}$ Research Scientist, Flow Physics and Control Branch, Senior Member AIAA

$\S$ Research Scientist, Flow Physics and Control Branch, Associate Fellow AIAA

『Graduate Student, Department of Aerospace Engineering, Student Member AIAA
} 


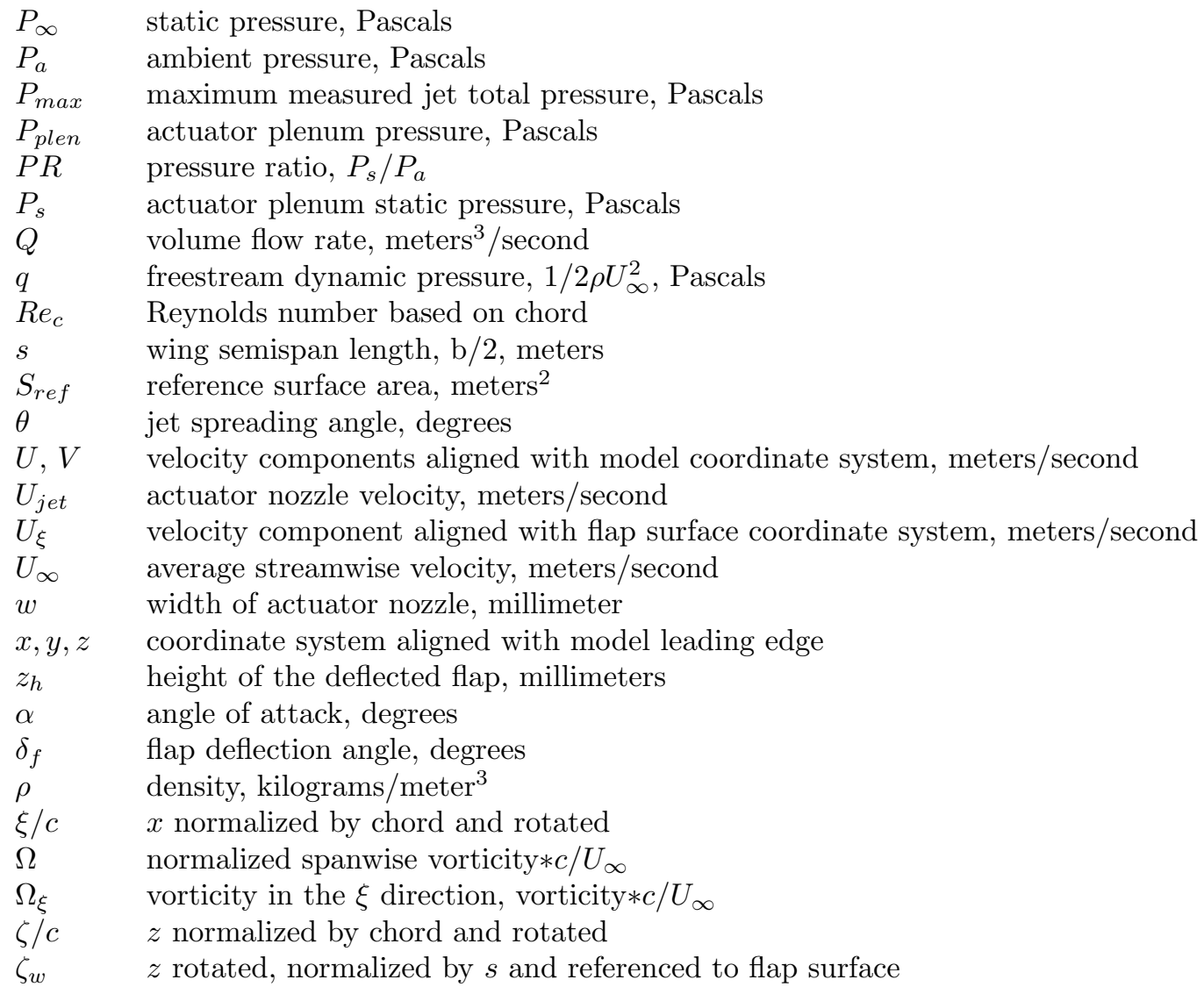

\section{A. Abbreviations}

2D two dimensional

3D three dimensional

AFC active flow control

BART Basic Aerodynamics Research Tunnel

CD convergent-divergent

CRM Common Research Model

LaRC Langley Research Center

PIV Particle Image Velocimetry

SLA stereolithography

TE trailing edge

SWJ sweeping jet

\section{Introduction}

In this experiment, AFC from the flap shoulder of an unswept, semispan wing model with a simplehinged, trailing edge flap is investigated because of the potential application of AFC technology to a high-lift system. McLean et al. ${ }^{1}$ studied applications of the technology on a commercial transport and found that the use of AFC on a high-lift system with a simple-hinged flap could be beneficial due to the reduction in part count and weight. Based on the findings of McLean et al., ${ }^{1}$ an AFC-enabled high-lift system might require a trailing edge flap deflection much larger than that required for the Fowler flap found on most conventional high-lift systems. For this reason, we are applying AFC for trailing edge flap deflections ranging from $20^{\circ}$ to $60^{\circ}$. A new trailing edge flap, which increases the maximum flap deflection from $40^{\circ}$ to $60^{\circ}$ on the NACA 0015 semispan wing used in previous AFC studies ${ }^{2-4}$ was built for the study. The flap deflection range of 
this study enables the evaluation of AFC in moderate to massively separated flowfields.

Renewed interest in AFC for high-lift applications by the NASA Advanced Air Transport Technology (AATT) Project motivated the current study. Recent successful AFC demonstrations ${ }^{5-7}$ using sweeping jet (SWJ) actuators have provided additional motivation for evaluating the ability of the technology to augment lift. Most prior high-lift AFC applications using simple-hinged flaps with deflections that exceeded $40^{\circ}$ were performed using zero-net-mass-flux (ZNMF) actuators. Nagib et al. ${ }^{8}$ performed AFC studies using ZNMF actuators on an airfoil with flap deflections up to $60^{\circ}$. They found that the unsteady excitation produced by the ZNMF actuators was unable to reattach the separated flow to the flap at $\delta_{f}=60^{\circ}$; however, the excitation increased lift due to an increase in circulation that was most prominent upstream of the excitation location. Similar results were obtained by Melton et al. ${ }^{9}$ when applying ZNMF actuation on the flap of a supercritical airfoil. The authors noted that applying AFC in the highly curved flap shoulder region at larger flap deflections was challenging due to the sensitivity of AFC to the actuation location and possible curvature effects. In the current study, two pneumatic actuation methods are investigated. One method is discrete steady jet actuation produced by convergent-divergent (CD) nozzles, which are similar to the actuators used in the computational study of Hartwich et al., ${ }^{10}$ and the other is SWJ actuation. ${ }^{11,12}$ The jets produced by these two excitation methods generate streamwise vortices that may enhance mixing in the boundary layer thereby controlling separation while the spanwise uniform ZNMF excitation used in References 8 and 9 generated spanwise coherent vortices that were believed to enhance mixing, making it an efficient method of separation control.

This small scale experiment at relatively low Reynolds numbers $\left(R e_{c} \leq 1.0 \times 10^{6}\right)$ is part of a larger project focused on developing an AFC-enabled high-lift system. The flap deflection range of the current investigation covers the range being proposed for a larger scale, wind tunnel experiment on a high-lift version of the Common Research Model (CRM) ${ }^{13}$ The goal of the small-scale experiments is to investigate methods to improve the input power requirements of the AFC system that will be evaluated on the high-lift CRM. In this paper, we employ surface oil flow visualization and off-body measurements using Particle Image Velocimetery (PIV) to investigate the interaction between the SWJ excitation and the shear layer associated with the flow separation. These results aid in understanding this interaction as the adverse pressure gradient, and thus the degree of separated flow on the flap, is increased with flap deflection. Previous studies ${ }^{4}$ showed that the SWJ actuator geometry, size, and spacing tested are effective at controlling separation on the NACA 0015 wing for flap deflections up to $40^{\circ}$. The results obtained using the SWJ excitation are compared to those obtained using steady jet excitation. The steady jet actuators have the same nozzle dimensions and spacing as the SWJ actuators and were located at the flap shoulder.

In addition to the two-dimensional (2D) and three-dimensional (3D) PIV results, force and moment data and surface static pressure data provide a quantitative measure of the overall benefits due to AFC. Benchtop actuator characterization results describing the flowfields produced by the two actuation methods are included, enabling a comparison of the two jet types.

\section{Experiment Description}

\section{A. Wind Tunnel Description}

The experiments were conducted in the NASA Langley Research Center (LaRC) Basic Aerodynamics Research Tunnel (BART) depicted in Fig. 1. The open-circuit tunnel has an 11:1 contraction ratio and a test section that is $0.71 \mathrm{~m}$ high by $1.02 \mathrm{~m}$ wide by $3.05 \mathrm{~m}$ long. The BART is used primarily as a flow physics research facility; therefore, it has the instrumentation and optical access needed for measurement techniques such as Laser Doppler Velocimetry and PIV. The maximum velocity of the tunnel is approximately $60 \mathrm{~m} / \mathrm{s}$ and we tested at speeds up to $35 \mathrm{~m} / \mathrm{s}$.

\section{B. Model Description}

The model tested is a $0.305 \mathrm{~m}$ chord (c), $0.610 \mathrm{~m}$ semipan (s) wing with an NACA 0015 cross-section that was built for sidewall AFC testing in the BART facility. ${ }^{2,14,15}$ In addition to the modified spar described in Ref. 3, a new $0.610 \mathrm{~m}$ span, $30 \%$ chord trailing edge flap (enabling flap deflection angles, $\delta_{f}$, from $-10^{\circ}$ to $60^{\circ}$ in $10^{\circ}$ increments) was used (Fig. 2(a)). The model has four rows of streamwise pressure taps at spanwise $(y / s)$ locations of $0.17,0.50,0.83$, and 0.99 and four rows of spanwise pressure taps at streamwise $(x / c)$ locations of $0.0050,0.30,0.77$, and 1.0. Transition dots with a height of $0.2 \mathrm{~mm}$, located approximately 1.14 


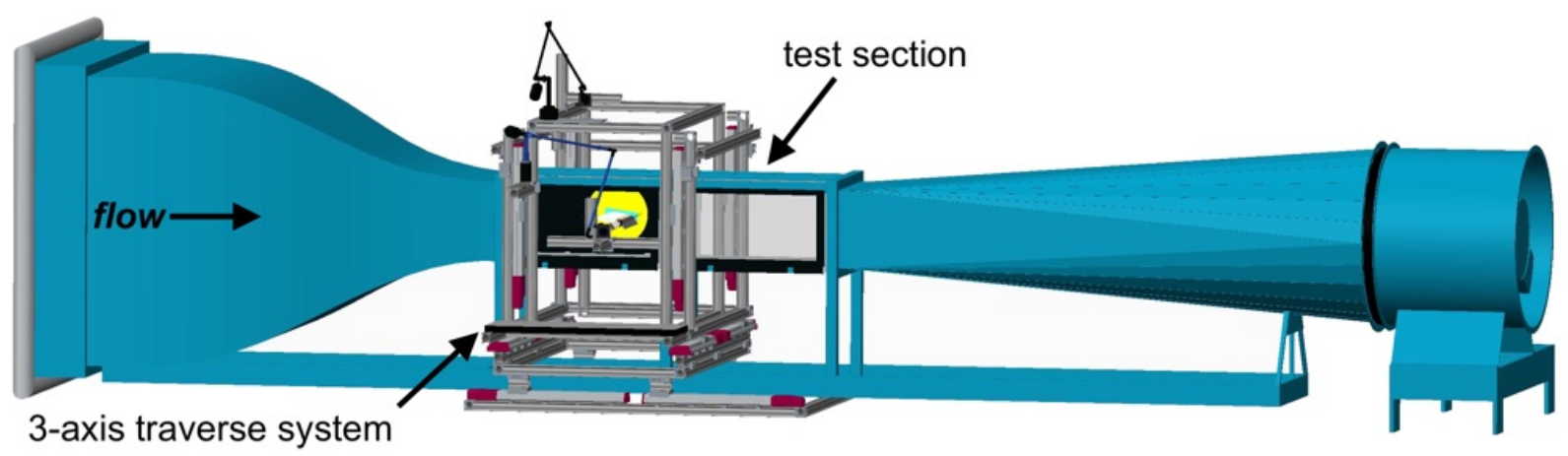

Figure 1. CAD image of the Basic Aerodynamics Research Tunnel (BART).

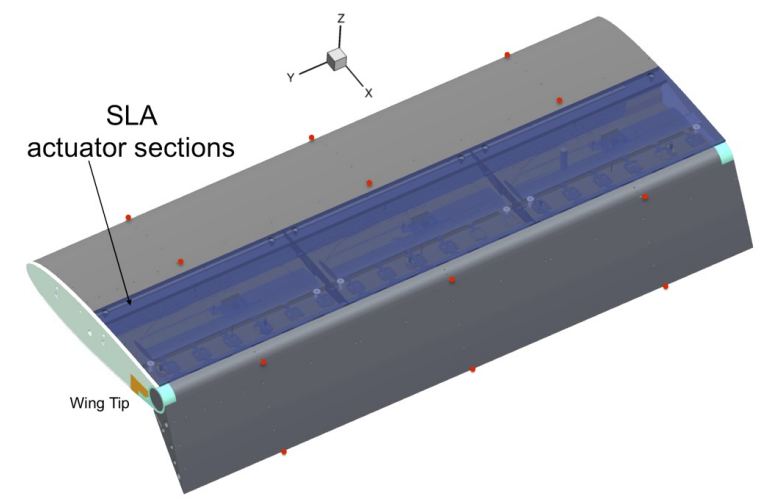

(a) NACA 0015 wing model.

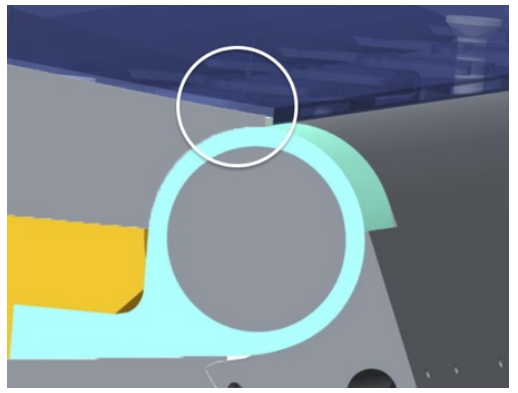

(b) Surface discontinuity at outboard edge of wing. White circle shows region where step is present.

Figure 2. CAD image of an NACA 0015 semispan wing model. The red circles represent unsteady pressure orifice locations. 
$\mathrm{cm}$ downstream of the leading edge, were applied to the upper surface of the model to minimize Reynolds number effects over the freestream velocity and flap deflection ranges of this investigation. The model has a leading edge excitation slot, which was unused but left open during this test, that promotes transition. Primer and flat black paint with a total thickness of $0.08 \mathrm{~mm}$ were added to the model surface to minimize laser reflections during the PIV portion of the test. A 5-component, external strain gauge balance was used to measure forces and moments on the model. The balance axial and yawing moment limits prevented testing at high angles of attack and high dynamic pressures.

\section{AFC Actuators}

The SWJ and CD actuator modules, manufactured using stereolithography (SLA), were built in three sections and located upstream of the flap shoulder as shown in Fig. 2(a). Each section was $0.20 \mathrm{~m}$ wide. The nozzle size of all actuators was $1 \mathrm{~mm}$ high $(h)$ by $2 \mathrm{~mm}$ wide $(w)$. Six actuators, spaced $3.3 \mathrm{~cm}$ apart, were in each SLA flap section. In preparation for a high Reynolds number AFC experiment, ${ }^{16}$ this SWJ actuator configuration was previously used to effectively control separation ${ }^{4}$ at flap deflection angles up to $40^{\circ}$. Unlike the experiments described in Refs. 3 and 4, the actuators were installed so that the outer mold line of the model was slightly modified (Fig. 2(b)). We selected this installation because of the improved spanwise flow uniformity with SWJ AFC compared to the results obtained using the recessed actuator installation. ${ }^{4}$ The actuator jet exited the model at the flap shoulder and was nearly tangential to the flap surface when the flap deflection angle was $0^{\circ}$.

Each of the three actuator sections was independently controlled by an electronic pressure regulator. Thermal mass flow meters in the three air supply lines were used to measure mass flow rate and each actuator plenum was instrumented with a static pressure orifice and a thermocouple for pressure and temperature measurements, respectively, which were used to compute density. The jet velocity is defined by Eq. 1, where, $A_{j e t}$ is the actuator nozzle area, $2 \mathrm{~mm}^{2}$, and density is computed using isentropic relations. $C_{\mu}$ is defined by Eq. 2. Several recent SWJ actuator AFC studies ${ }^{5,6}$ have used similar definitions for $C_{\mu}$. Variations of this definition have also been used where density, $\rho$, is assumed to be constant.

$$
\begin{aligned}
U_{j e t} & =\frac{\dot{m}}{\rho n_{j e t} A_{j e t}} \\
C_{\mu} & =\frac{\dot{m} U_{j e t}}{q S_{r e f}}
\end{aligned}
$$

\section{Particle Image Velocimetry}

A 2D PIV system comprised of a pulsed Nd:YAG laser operated at $10 \mathrm{~Hz}$, a CCD camera with a 2048 pixel $\mathrm{x}$ 2048 pixel sensor, a camerlink interface, and acquisition software written by Wernet ${ }^{17}$ was used to acquire the streamwise PIV data. A 3D system, using similar hardware, was used for the spanwise PIV measurements. A $105 \mathrm{~mm}$ micro lens was used with the streamwise camera, and the field of view was $158 \mathrm{~mm} \times 158 \mathrm{~mm}$ for the data obtained at $\delta_{f}=20^{\circ}$ and $\delta_{f}=40^{\circ}$ and $168 \mathrm{~mm} \times 168 \mathrm{~mm}$ for the data obtained at $\delta_{f}=60^{\circ}$. Two, $150 \mathrm{~mm}$ macro lenses were used with the spanwise PIV cameras and the field of view was $127 \mathrm{~mm} \mathrm{x}$ $127 \mathrm{~mm}$ for the $\delta_{f}=40^{\circ}$ measurements, $91 \mathrm{~mm} \times 91 \mathrm{~mm}$ for the $\delta_{f}=60^{\circ}$ SWJ actuator measurements, and $95 \mathrm{~mm} \times 95 \mathrm{~mm}$ with the CD actuator. Laser light guide arms and commercial off-the-shelf light sheet forming optics modules were used to generate both the streamwise and spanwise lightsheets. The data were processed using commercially available PIV analysis software. ${ }^{18}$ The interrogation window was 24 pixels $\mathrm{x}$ 24 pixels with an overlap of $50 \%$. The streamwise camera, located on the side of the tunnel opposite the balance, was rotated so that the flap surface was horizontal in the camera view. Most of the streamwise PIV data were acquired at $y / s=0.504$ (to the right of the model centerline when looking downstream). The two-dimensional streamwise and stereoscopic spanwise PIV systems (lightsheet forming optics and cameras) were mounted on the three-axis traverse system that surrounds the BART facility to simplify calibration and data acquisition at multiple $x$ and $y$ locations. The flowfield was seeded with 1 micron particles that were produced by a theatrical smoke generator. In most cases, at least 600 image pairs were used to compute the mean values for the 2D PIV results and 800 image pairs were typically used for the 3D PIV results. 


\section{Actuator Characterization}

Both SWJ and steady jets (CD) actuators (Fig. 3) that rely on pneumatic input were used for this study because of their flow control authority. The selected SWJ actuator geometry (Fig. 3(b)) was used previously on this model ${ }^{4}$ to control separation and shown to be more efficient in terms of power than the two other SWJ actuators geometries included in the study. The diffuser length of the SWJ actuator was extended to take advantage of the increase in sweep or spreading angle due to jet attachment to the diffuser walls referred to as a Coanda extension in Ref. 19. The CD actuator (Fig. 3(a)) was investigated because the computational studies performed by Hartwich et al. ${ }^{10}$ showed that this type of actuator was also effective at controlling sep-

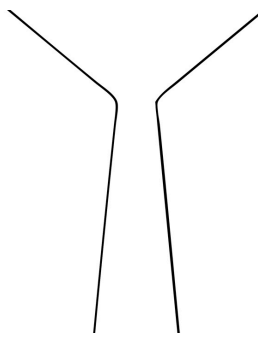

(a) CD.

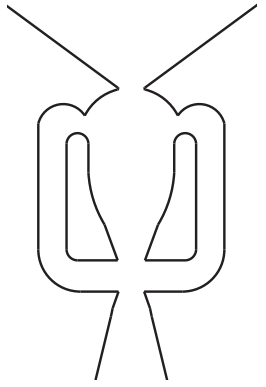

(b) SWJ.

Figure 3. Actuator geometries. aration. Additionally, the CD actuator is easier to model computationally and could be easier to manufacture (depending on the method selected) than the SWJ actuator used in this study. It should be noted that we did not optimize the internal geometry of the CD actuator.

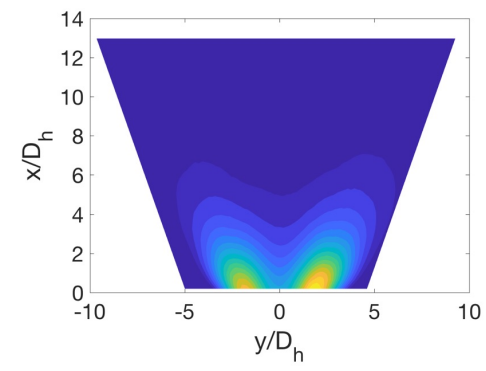

(a) SWJ, $P R=1.6$.

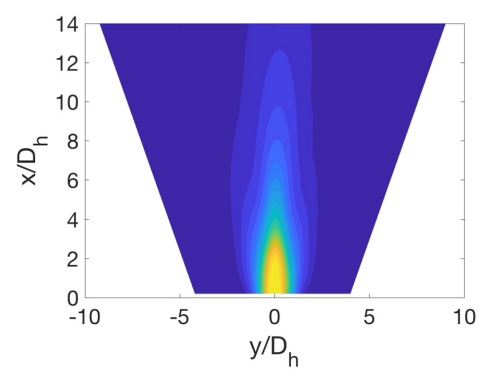

(d) $\mathrm{CD}, P R=1.5$.

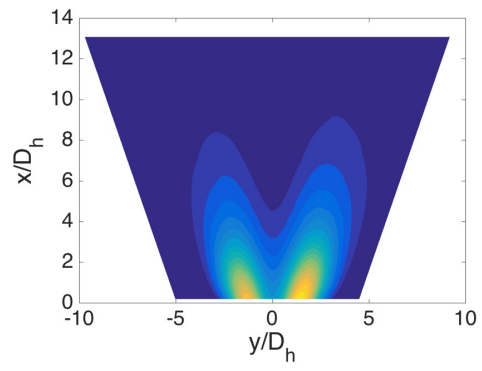

(b) SWJ, $P R=2.3$.

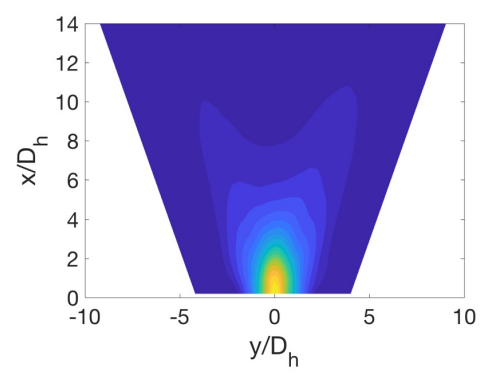

(e) $\mathrm{CD}, P R=2.3$.

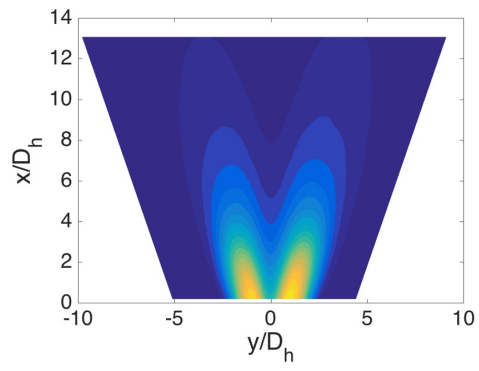

(c) SWJ $P R=3.1$.

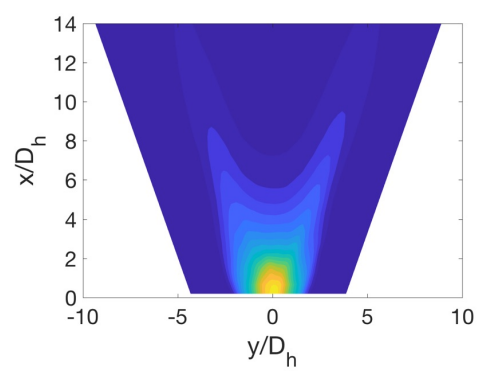

(f) $\mathrm{CD}, P R=3.1$.

Figure 4. Total pressure contours of the SWJ and CD actuator geometries.

The internal flowfields of several fluidic oscillators have been investigated both experimentally and numerically. ${ }^{20-22}$ The purpose of the benchtop characterization studies was to document the jets produced by the relatively small actuators used for this set of experiments. The unsteady pressure probe described in Ref. 4 was used to characterize the individual actuators built for the benchtop studies. Figure 4 shows the mean total pressure contours for both the SWJ and CD actuators. We scaled the data to fit the pressure range of each contour; therefore, Table 1 includes the maximum mean pressure measured in each jet flowfield normalized by the actuator plenum pressure $\left(P_{\max } / P_{\text {plen }}\right)$. Although not shown here, we also used nonintrusive methods such as schlieren imaging and surface oil flow to verify the trends obtained from the unsteady pressure probe measurements. The SWJ actuator produces a spatially oscillating jet that spends more time on each side of the diffuser walls due to jet attachment to one of the two internal Coanda surfaces 
of the actuator. Hence, two jet cores are evident in the mean pressure contours of Figs. 4(a) to 4(c). The data also show that the jet sweep angle is reduced with increasing $P R$.

The oscillation frequency of the SWJ is dependent on the actuator geometry and the mass flow supplied to the actuator. Figure 5 presents the frequency characteristics of the SWJ actuator as the actuator input pressure (and thus mass flow rate) is varied. The data show that the operating frequency of the actuator is approximately $1500 \mathrm{~Hz}$ at $P R=3.9$. This frequency value is roughly an order of magnitude larger than the natural instabilities in the flowfield. Also, each SWJ actuator operates independently resulting in a random phase difference between adjacent actuators.

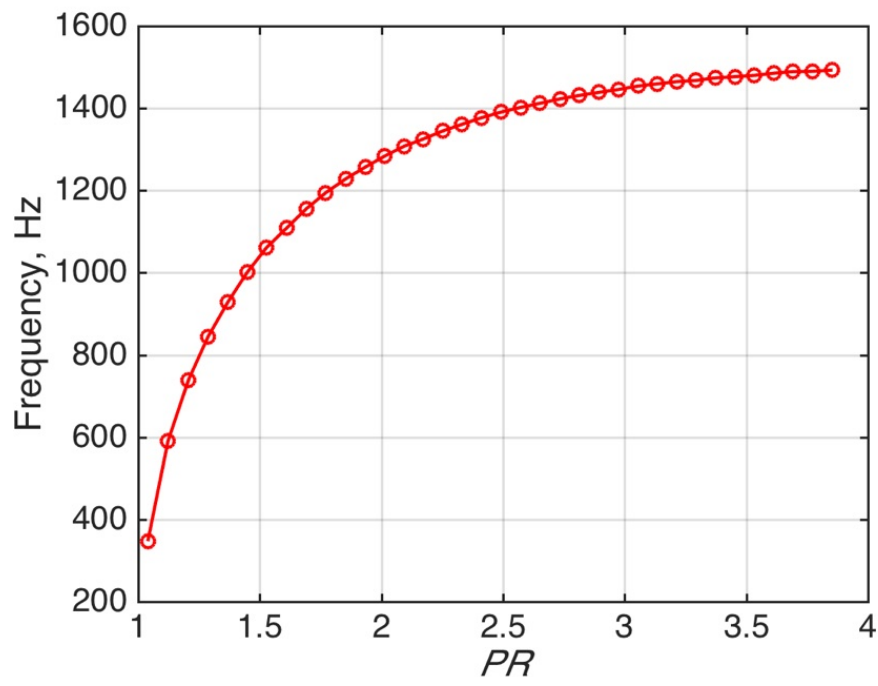

Figure 5. Actuator frequency as a function of $P R$ for the SWJ actuator.

Table 1. SWJ and CD Actuator Characteristics.

\begin{tabular}{|c|c|c|c|c|c|}
\hline \hline \multicolumn{3}{|c|}{ SWJ } & \multicolumn{3}{c|}{ CD } \\
\hline \hline$P R$ & $P_{\text {max }} / P_{\text {plen }}$ & $\dot{m}, \mathrm{~g} / \mathrm{s}$ & $P R$ & $P_{\max } / P_{\text {plen }}$ & $\dot{m}, \mathrm{~g} / \mathrm{s}$ \\
\hline 1.6 & 0.68 & 0.60 & 1.5 & 0.98 & 0.63 \\
\hline 2.3 & 0.61 & 0.94 & 2.3 & 0.92 & 1.08 \\
\hline 3.1 & 0.60 & 1.34 & 3.1 & 0.82 & 1.53 \\
\hline
\end{tabular}

The CD actuator geometry produces a steady, straight jet as shown in Figs. 4(d) to 4(f). At the lowest $P R$ presented (Fig. 4(d)), the jet extends farther in the $x / D_{h}$ direction than the oscillating jet produced by the SWJ actuator due to conservation of mass, and the maximum mean pressure, measured at the jet exit, is comparable to the actuator plenum pressure. The jet spreading angle almost doubles for the two higher pressure ratio values due to a supersonic expansion of the flow. Accompanying this expansion is a reduction in $P_{\text {max }} / P_{\text {plen }}$. The CD actuator has a higher mass flow rate for a fixed $P R$ compared to the SWJ actuator (Table 1).

\section{AFC Results}

As mentioned previously, this model has been used to study the effectiveness of various SWJ configurations. $^{3,4}$ However, previous investigations were limited to $\delta_{f} \leq 40^{\circ}$. We will begin by presenting results comparing the baseline flowfields with $\delta_{f}=20^{\circ}, 40^{\circ}$, and $60^{\circ}$. AFC results are then presented showing the lift increment, $\Delta C_{L}$, that can be obtained as $\delta_{f}$ is increased from $20^{\circ}$ to $60^{\circ}$. PIV results at a single spanwise location $(y / s=0.504)$ are presented to illustrate the changes due to AFC via SWJ and CD actuation. Sur- 
face flow visualization results are also presented to provide a more global view of the effect of AFC. Finally, spanwise PIV planes are presented and discussed showing the interaction of the SWJ and CD actuation with the separated shear layer.

\section{A. Baseline Flowfield}

Streamlines computed from mean PIV data for the baseline flowfield at the three flap deflections investigated are presented in Fig. 6 . The PIV data are presented so that the trailing edge flap of the model is horizontal to maximize the field of view over the flap. The data show that the size of the separated region on the flap increases with $\delta_{f}$. The PIV results also indicate that the positive vortices shed from the lower surface at the TE increase in size and circulation level as the flap deflection increases. The center of the time-averaged negative vortex consistently moves upstream. The shedding frequency of these vortices was measured by the unsteady pressure transducers in the trailing edge (TE) flap of the model. Fig. 7(a) presents spectra for the three flap deflection angles, and Fig. 7(b) presents spectra for $\delta_{f}=60^{\circ}$ at various Reynolds numbers. The shedding frequency, when $\delta_{f}=60^{\circ}$, is detected not only by the TE unsteady pressure transducers but also by additional transducers located upstream of the TE due to the shed vortices being closer to the flap surface with increasing $\delta_{f}$.

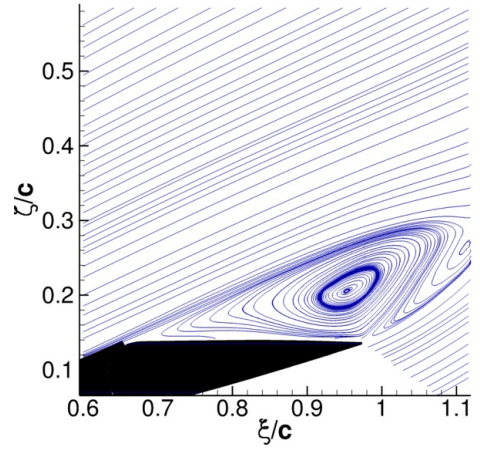

(a) $\delta_{f}=20^{\circ}$.

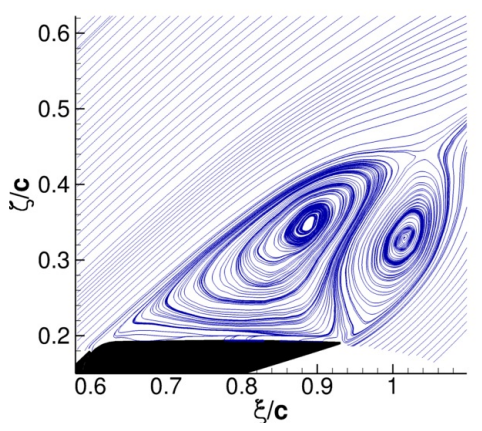

(b) $\delta_{f}=40^{\circ}$.

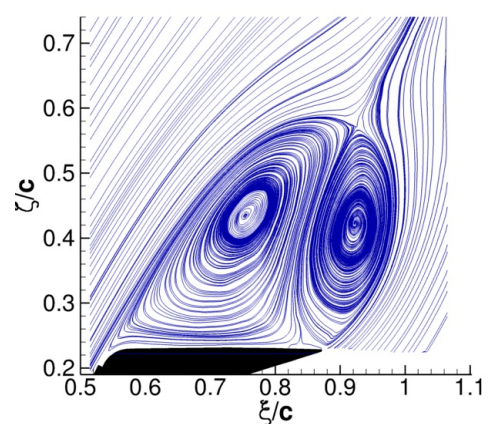

(c) $\delta_{f}=60^{\circ}$.

Figure 6. Streamlines from PIV data for the baseline flowfield, $\alpha=8^{\circ}, y / s=0.504$.

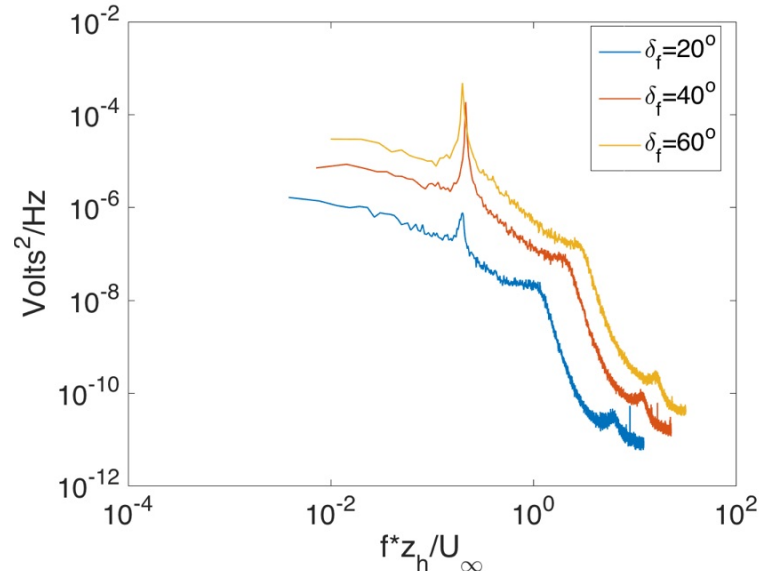

(a) $\delta_{f}$ varied, $R e_{c}=500,000$.

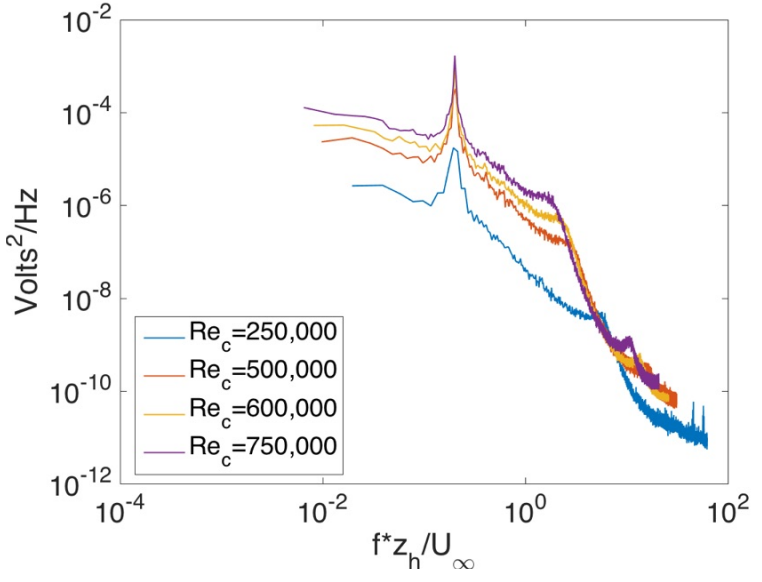

(b) $R e_{c}$ varied, $\delta_{f}=60^{\circ}$.

Figure 7. Spectra from flap trailing edge unsteady pressures. 


\section{B. SWJ and CD Actuators}

In contrast to the ZNMF actuators previously used with this model, the SWJ actuators have the control authority needed to attach the flow to the flap. For this reason, our approach to determining the upper limit of $C_{\mu}$ for the two lower flap deflection cases $\left(\delta_{f}=20^{\circ}\right.$ and $\left.\delta_{f}=40^{\circ}\right)$ was based on the pressure recovery generated by the excitation. The pressure recoveries for these two $\delta_{f}$ cases were deemed acceptable when the trailing edge pressure $C_{p, T E}$, was 0 . Figure 8 presents the streamwise $C_{p}$ distributions from the midspan location, $y / s=0.5$ and the spanwise $C_{p, T E}$ distributions over the flap deflection range investigated with varying levels of AFC. The baseline, separated flow in Fig. 8(a) has a plateau in $C_{p}$ on the flap. With SWJ actuator control introduced at the flap shoulder, a pressure recovery is apparent on the flap and $C_{p, T E}$ is near 0 when $C_{\mu}=0.89 \%$. The spanwise $C_{p}$ distribution (Fig. 8(b)) along the TE of the flap indicates that the pressure recovery obtained at $y / s=0.5$ with $\delta_{f}=20^{\circ}$ is achieved over the majority of the span of the wing. The juncture vortex present at the inboard region of the model $(y / s<0.1)$ reduces the $C_{p}$ values inboard and the tip vortex reduces the outboard $(y / s>0.85) C_{p}$ values. The increase in circulation upstream of the SWJ excitation location and the increase in the suction peak at the flap shoulder contribute to the lift increment when AFC is applied. Similar results are presented in Figs. 8(c) and 8(d) with $\delta_{f}=40^{\circ}$, although $C_{\mu}=2.65 \%$ is required to obtain $C_{p, T E}=0$. The streamwise $C_{p}$ distributions of Fig. 8(c) indicate that there is a larger suction peak at the flap shoulder due to the larger value of $C_{\mu}$ required to attach the flow and an increase in circulation upstream of the SWJ excitation location. The $C_{p, T E}$ distribution in Fig. 8(d) is not as uniform as that obtained with $\delta_{f}=20^{\circ}$ (Fig. 8(b)). The nonuniformity is observed at both input levels shown in Fig. 8(d) with control appearing less effective near $y / s=0.4$ with $C_{\mu}=2.65 \%$. SWJ AFC introduced at the flap shoulder of the $\delta_{f}=60^{\circ}$ model configuration does not produce the pressure recovery obtained when applying AFC to the $\delta_{f}=20^{\circ}$ and $\delta_{f}=40^{\circ}$ configurations. The streamwise $C_{p}$ distribution with SWJ actuation shown in Fig. 8(e) indicates that $C_{\mu}=4.58 \%$, increases circulation and the suction peak at the flap shoulder. However, the suction peak at the flap shoulder is less than that obtained when applying SWJ actuation to the $\delta_{f}=40^{\circ}$ configuration (Fig. 8(c)). The TE $C_{p}$ distribution indicate that the $C_{p}$ value obtained at the midspan location is obtained over the fraction of the span that is not influenced by the wing tip and juncture vortices. The wing tip vortex is barely discernible in the baseline $C_{p}$ distribution at the TE (Fig. 8(f)). SWJ actuation at this flap deflection causes the tip vortex to influence a larger portion of the outboard span of the model as compared to the lower $\delta_{f}$ cases presented. Figs. $8(\mathrm{~g})$ and $8(\mathrm{~h})$ present the $C_{p}$ distributions when using the CD actuator to control flow separation with $\delta_{f}=60^{\circ}$. The streamwise $C_{p}$ distributions of Fig. $8(\mathrm{~g})$ indicate that the CD actuation produces a smaller suction peak at the flap shoulder, less circulation upstream of the actuation location, and less pressure recovery on the flap when compared to the results obtained with the $\delta_{f}=60^{\circ}$ SWJ AFC (Fig. 8(e)). The spanwise $C_{p}$ distribution of Fig. $8(\mathrm{~h})$ shows that the tip vortex with $\mathrm{CD}$ actuation influences the outer $22 \%$ of the trailing edge wing span.

Figure 9 presents the increment in lift as a function of mass flow coefficient, $C_{q}$, for the three flap deflections studied using SWJ actuators for control. Data are also included comparing the SWJ excitation to the $\mathrm{CD}$ excitation at the maximum flap deflection, $\delta_{f}=60^{\circ}$. The maximum $C_{q}$ level presented for the $\delta_{f}=20^{\circ}$ and $\delta_{f}=40^{\circ}$ cases generated pressure recoveries resulting in $C_{p, T E}=0$. The data show that the SWJ actuators can be used to improve lift over the entire range of flap deflection angles studied. Above $C_{q}=0.1 \%$, the mass flow required to achieve a given lift increment increases with flap deflection angle when using SWJ excitation. The discrete steady jet excitation produced by the CD actuators becomes more effective above a $C_{q}$ level of $0.2 \%$. The unsteady pressure data for $\delta_{f}=60^{\circ}$ indicate that when $C_{q} \leq 0.09 \%$ for the SWJ actuator and $C_{q} \leq 0.2 \%$ for the CD actuator, the vortex shedding frequency is still present in the trailing pressure spectra, possibly explaining the change in slope for these two cases in Fig. 9. The effectiveness of the CD actuator is partly due to the jet expansion that occurs when the throat becomes sonic $(P R>2)$. At the highest $C_{q}$ level presented, there is a reduction in lift when using the CD actuators due to the flow being massively separated.

To obtain the global effects of actuation at $\delta_{f}=60^{\circ}$, flow visualization studies were performed using fluorescent oil flow. The oil flow is a mixture of 5 centistoke silicone and flouroescent pigment. Results from the oil flow visualization studies with $\delta_{f}=40^{\circ}$ were presented and discussed in Ref. 4 . The oil flow results in Ref. 4 were difficult to interpret due to the surface discontinuities produced by the hinges used with the segmented flap and the steps created by the tape covering the pressure orifices. The flap used for this study is continuous and contact paper was used to smooth the discontinuities due to the mylar tape that covered the flap pressure orifices. Oil was applied to the flap downstream of the actuator exit to minimize 


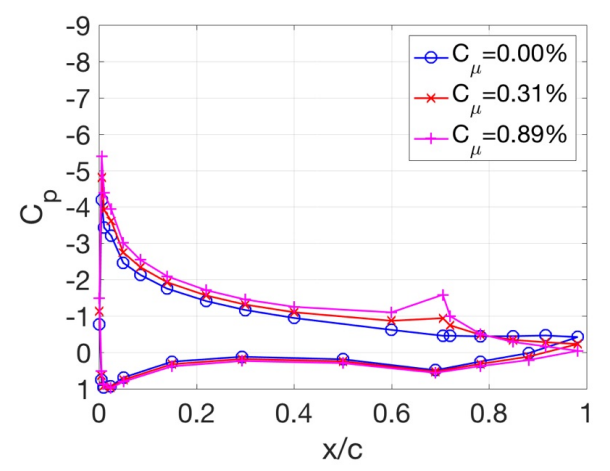

(a) $\delta_{f}=20^{\circ}, y / s=0.5$, SWJ.

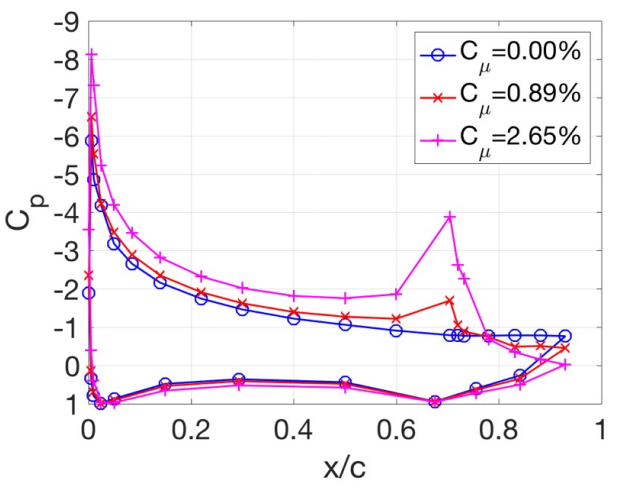

(c) $\delta_{f}=40^{\circ}, y / s=0.5$, SWJ.

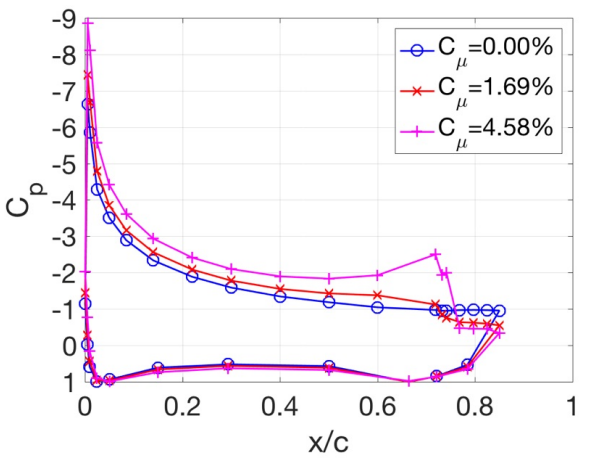

(e) $\delta_{f}=60^{\circ}, y / s=0.5$, SWJ.

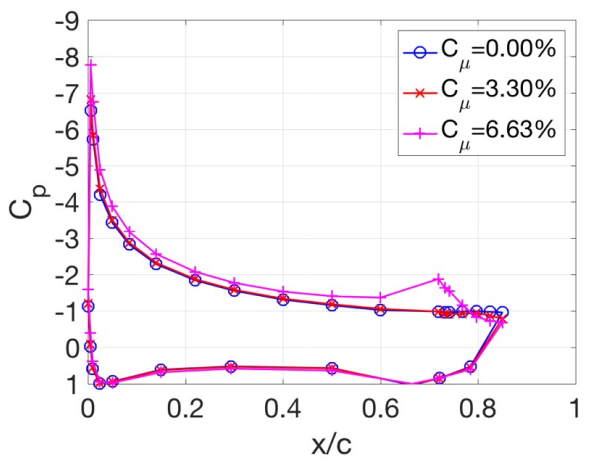

(g) $\delta_{f}=60^{\circ}, y / s=0.5$, CD.

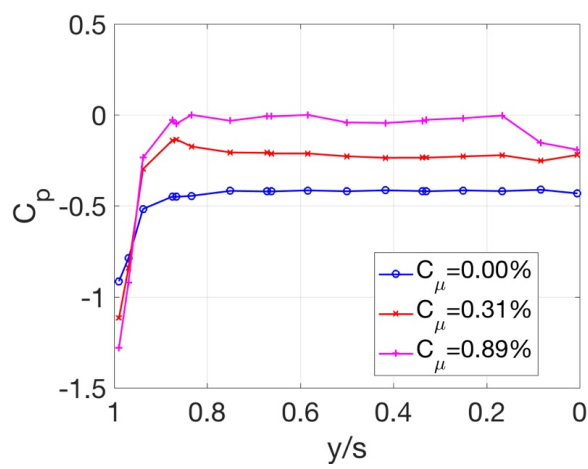

(b) $\delta_{f}=20^{\circ}$, TE, SWJ.

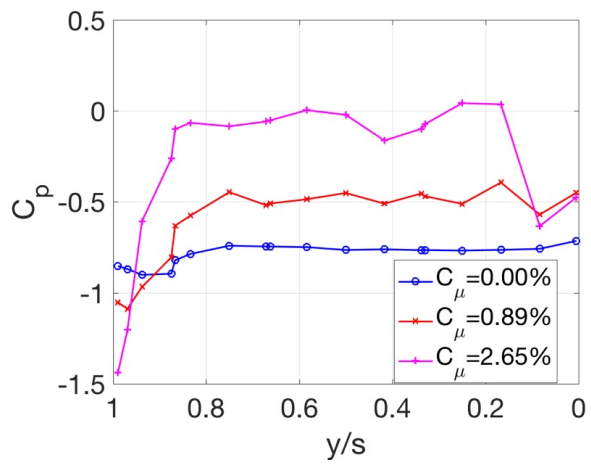

(d) $\delta_{f}=40^{\circ}$, TE, SWJ.

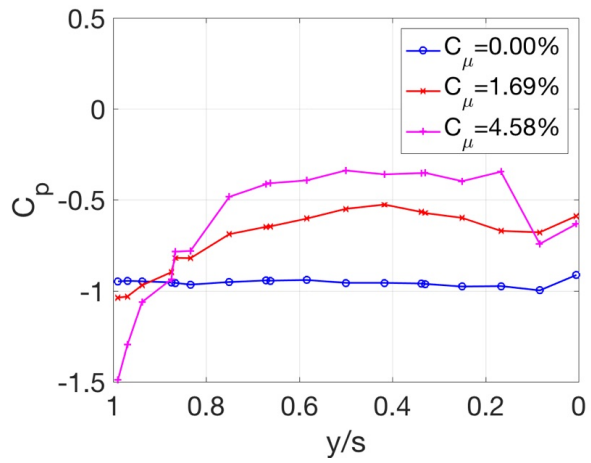

(f) $\delta_{f}=60^{\circ}, \mathrm{TE}, \mathrm{SWJ}$.

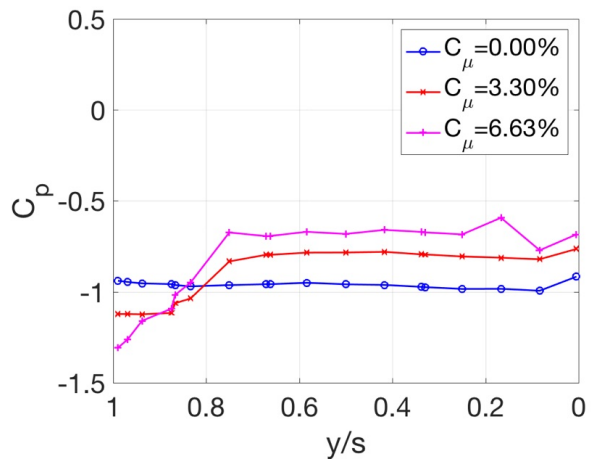

(h) $\delta_{f}=60^{\circ}, \mathrm{TE}, \mathrm{CD}$.

Figure 8. $C_{p}$ distributions at streamwise location $y / s=0.50$ and spanwise location, TE, $R e_{c}=500,000, \alpha=8^{\circ}$. 


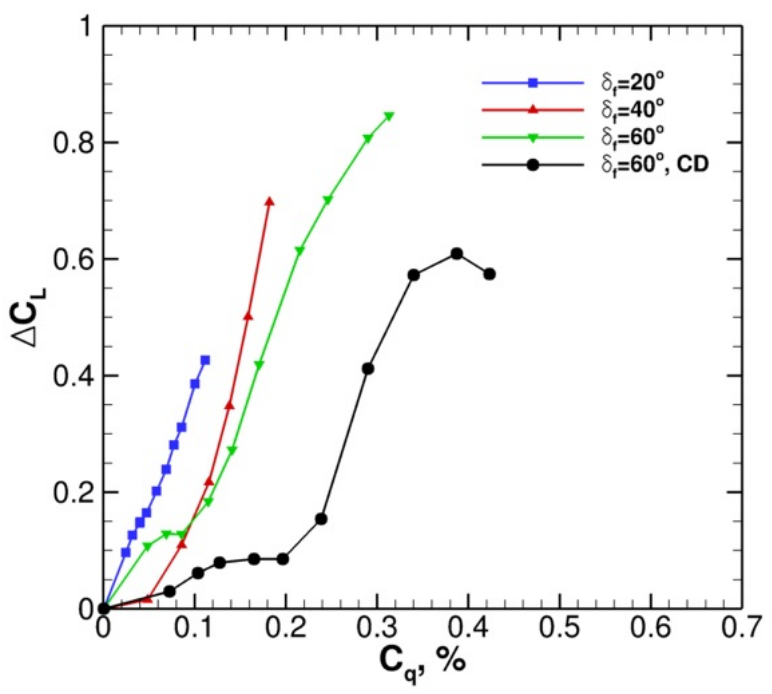

Figure 9. Lift Increment, $R e_{c}=500,000, \alpha=8^{\circ}$.

oil contaminating the actuator nozzles. Figures 10(a) and 11(a) show the oil flow patterns produced by the SWJ and CD actuators, respectively, when $U_{\infty}=0$. The images are cropped so that the flap is shown in the view with the right-hand side being the inboard side of the model and the bottom being the TE. The spatial oscillations of the SWJ actuators (Fig. 10(a)) cause them to have a larger spanwise coverage than the CD actuators (Fig. 11(a)). The regions between SWJ actuators, along the entire flap span, are denoted by dark lines due to the upwash generated by the counter-rotating vortices of adjacent SWJ actuators. In contrast, there are concentrations of undisturbed oil between CD actuators, indicating the CD actuator jets have a more localized effect near the leading edge of the flap. Also, the CD actuator jet cores are more prominent along the flap span in Fig. 11(a). Oil flow results with the CD actuators at $P R<2.0$ (not shown) indicate that the jets are narrower, consistent with the smaller jet spreading angle described in Section III.

Figures 10(b) and 10(c) present SWJ AFC oil flow results at $\mathrm{M}=0.07$. The presence of a wing tip vortex (left-hand side of images) and juncture vortex (right-hand side of images) is apparent by the spanwise flow patterns in the oil flow in these regions. The flap flowfields with $\delta_{f}=60^{\circ}$ and SWJ AFC (Figs. 10(b) and $10(\mathrm{c})$ ) are characterized by three dimensional flow indicating that the flow is not completely attached. Vortex pairs with their axes perpendicular to the flap surface are present near the jet exit at the boundary between SWJ actuators for both $C_{q}$ levels presented. These vortices are more pronounced for the higher $C_{q}$ case of Fig. 10(c), the flow over the flap is more uniform, and the counter-rotating streamwise vortices at the actuator interfaces extend further in the streamwise direction, indicative of more attached flow.

Figures 11(b) and 11(c) present oil flow results with $M=0.07$ using the $C D$ actuators. The results of Fig. 11(b) show that with crossflow the steady jet produced by the CD actuator at $P R=1.4$ generates a pair of vortices that are centered at the jet exit. The presence of the jet is noted in the oil by a dark line at the centerline of the each jet. Local regions of low speed, potentially separated flow are evident near the flap leading edge, between CD actuators in Fig. 11(c). The momentum introduced into the flowfield with $C_{q}=0.31 \%$ attaches the flow to the flap over a short streamwise distance. Flow separation is marked by the three-dimensionality of the flow that begins downstream of the flap leading edge.

Figure 12 presents vorticity contours and streamlines for the flow over the flap with $\delta_{f}=60^{\circ}$. As illustrated in the $C_{p}$ distributions of Fig. 8(e), the SWJ excitation is unable to completely reattach the flow to the flap. The vorticity contours for the case presented in Fig. 12(b) indicate that at the lowest $C_{q}$ level, the excitation elongates the separated region on the flap causing the recirculation region to appear larger than the baseline flowfield (Fig. 12(a)). When the mass flow rate is increased to $C_{q}=0.17 \%$ (Fig. 12(c)), the size of the recirculation region is reduced, accompanied by reattachment of the separated shear layer to the leading edge of the flap. Immediately downstream of the flap shoulder the flow separates. As mass 


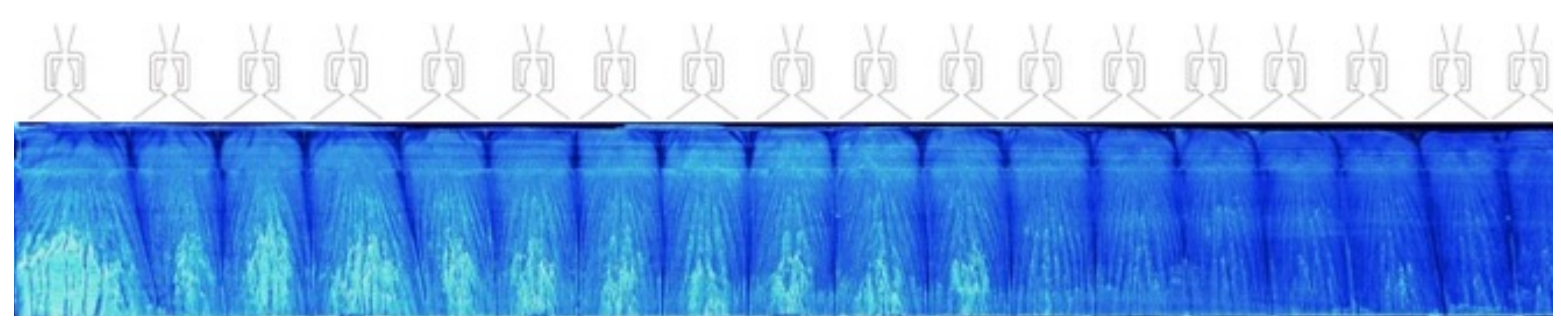

(a) $P R=2.0, M=0$.

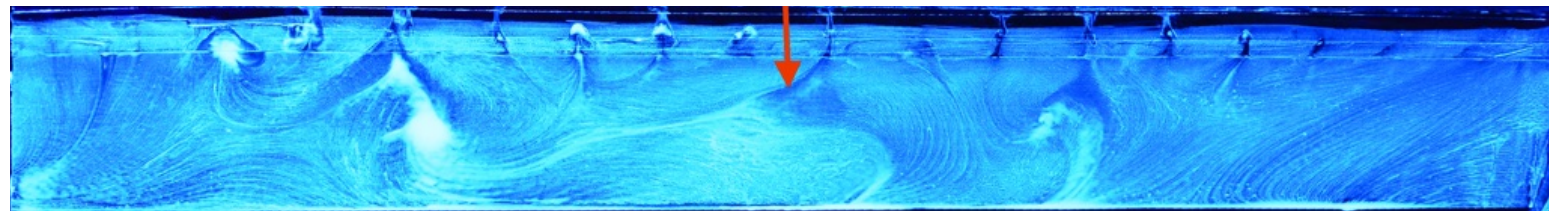

(b) $P R=1.5, C_{q}=0.14 \%, C_{\mu}=1.69 \%, M=0.07$.

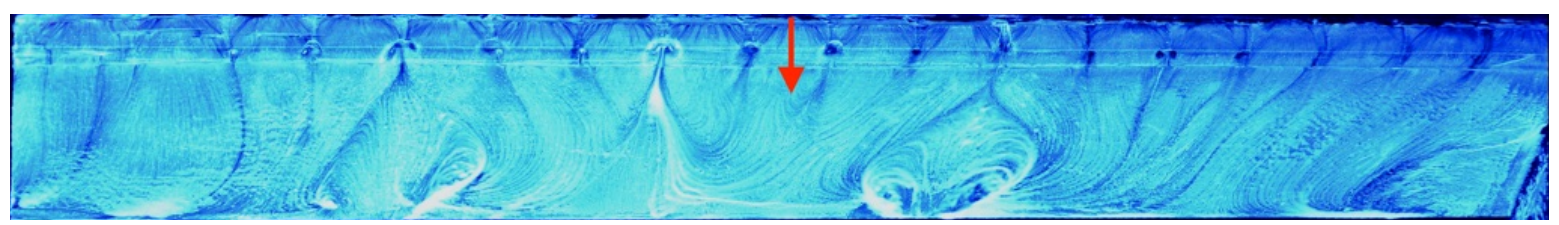

(c) $P R=2.0, C_{q}=0.22 \%, C_{\mu}=3.61 \%, M=0.07$.

Figure 10. Surface oil flow visualization using SWJ actuators. $R e_{c}=500,000, \delta_{f}=60^{\circ}$. Red arrows represent flow direction.

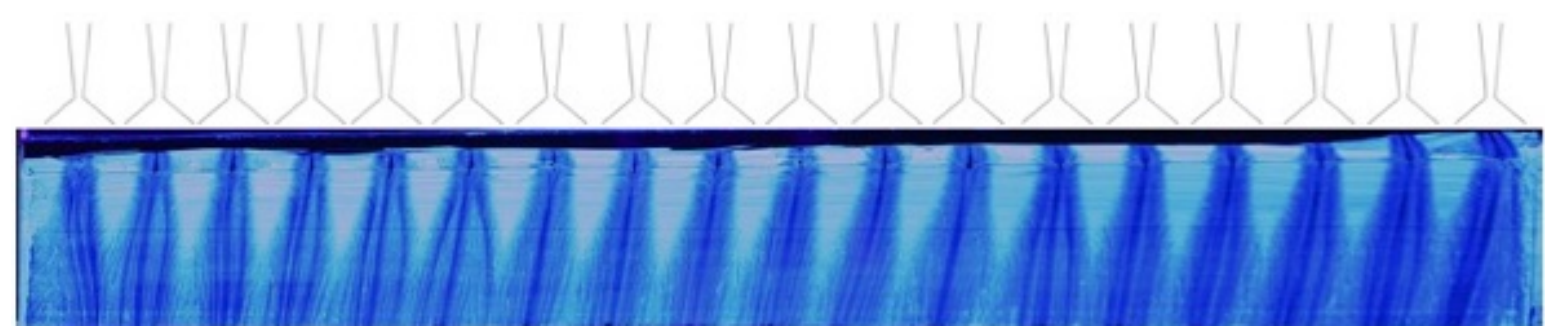

(a) $P R=2.0, M=0$.

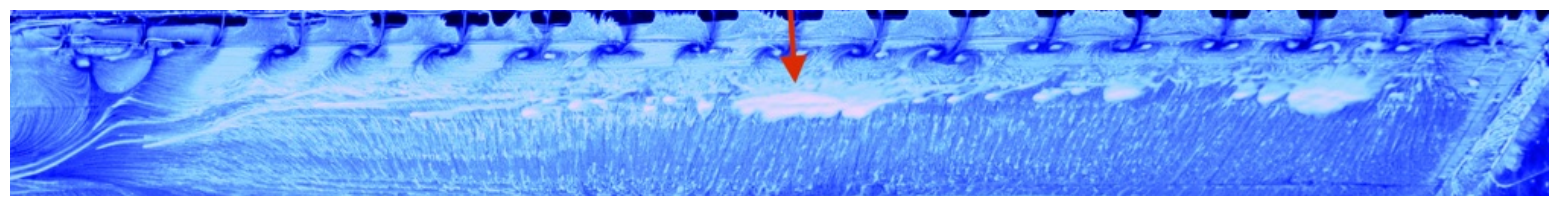

(b) $P R=1.4, C_{q}=0.17 \%, C_{\mu}=1.4 \%, M=0.07$.

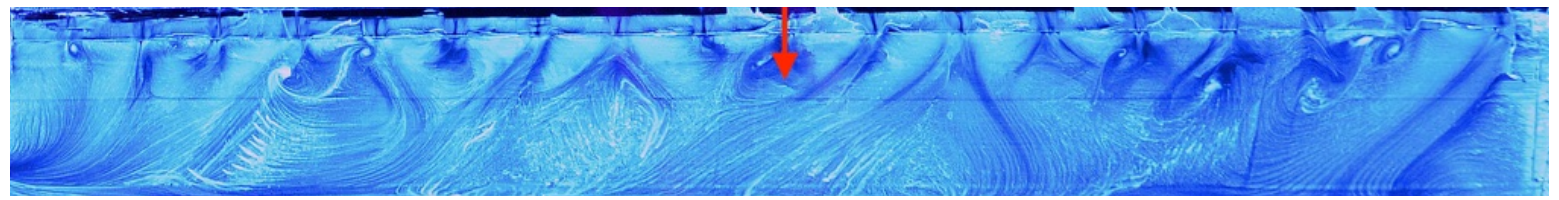

(c) $P R=2.0, C_{q}=0.31 \%, C_{\mu}=6.6 \%, M=0.07$.

Figure 11. Surface oil flow visualization using CD actuators. $R e_{c}=500,000, \delta_{f}=60^{\circ}$. Red arrows represent flow direction. 
flow is increased further (Fig. 12(d)), the excitation reduces the size of the recirculation region but appears to reattach only a portion of the initially separated shear layer above the flap. For mass flow levels above $C_{q}=0.17 \%$, the $C_{p}$ distributions (not shown) indicate that the addition of mass flow does not promote flow reattachment beyond the streamwise location shown in Fig. 12(d).

Vorticity contours are presented in Figs. 12(e) to 12(h) for the data obtained using the CD actuators to control separation. At a $C_{q}$ level of $0.17 \%$ (Fig. $12(\mathrm{f})$ ), the vorticity contours indicate that the CD excitation is altering the recirculation bubble and may explain why the vortex shedding frequency is the most dominant feature present in the TE unsteady pressure spectra. Increasing the CD excitation level to $C_{q}=0.29 \%$ (Fig. $12(\mathrm{~g})$ ) reduces the overall size of the separated region with some flow reattachment to the flap surface. In Fig. 12(h), the shear layer is completely separated from the flap shoulder due to the massively separated flow region. The larger $C_{L}$ values for the $C_{q}=0.42 \%$ results compared to the $C_{q}=0.29 \%$ results are due to an increase in circulation upstream of the actuation location.

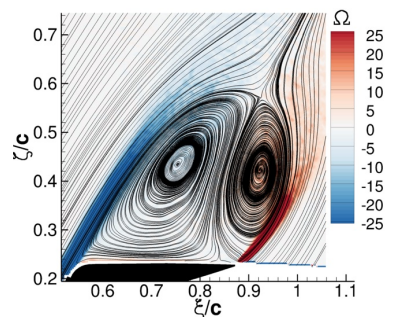

(a) SWJ, baseline.

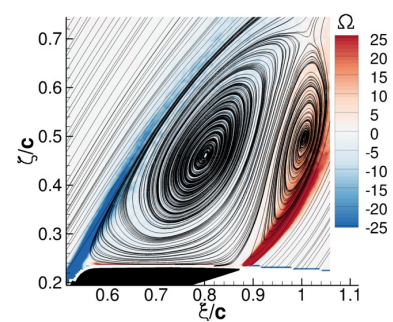

(b) SWJ, $C_{q}=0.12 \%$.

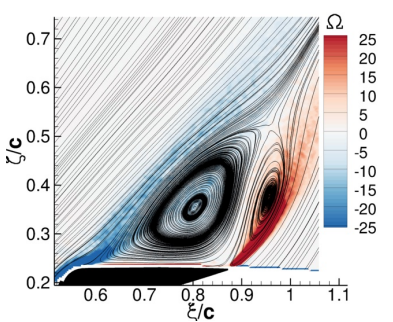

(c) SWJ, $C_{q}=0.17 \%$.

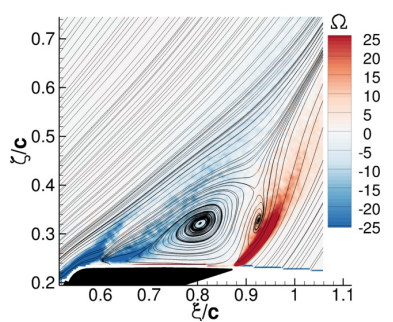

(d) SWJ, $C_{q}=0.22 \%$.

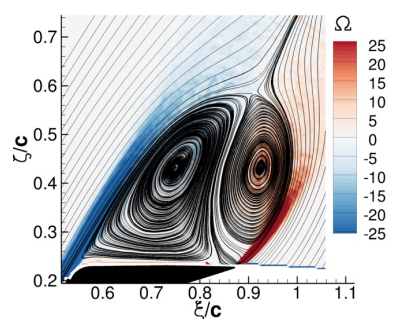

(e) CD, baseline.

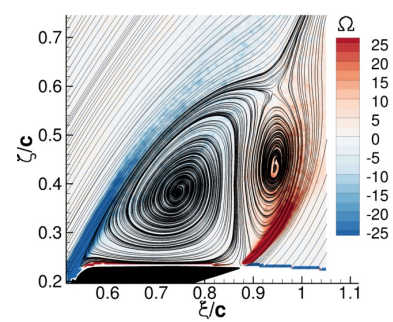

(f) $\mathrm{CD}, C_{q}=0.17 \%$.

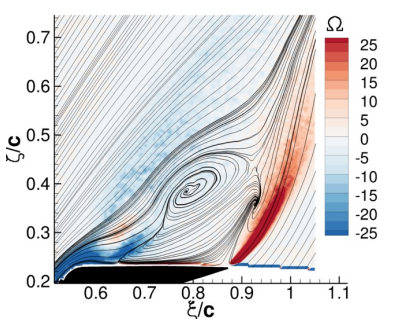

(g) CD, $C_{q}=0.29 \%$.

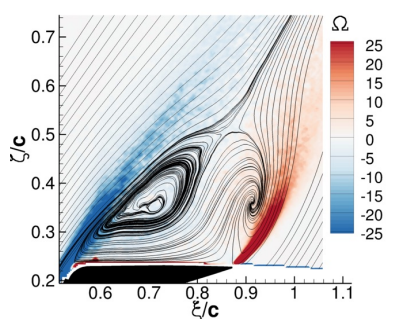

(h) $\mathrm{CD}, C_{q}=0.42 \%$.

Figure 12. PIV vorticity contours with streamlines for $y / s=0.504, \delta_{f}=60^{\circ}, \alpha=8^{\circ}$, and $\operatorname{Re}_{c}=500,000$.

\section{AFC-Interaction with Separated Shear Layer}

Spanwise PIV measurements were made at varying streamwise locations and analyzed to study the interaction between the excitations produced by the SWJ and CD actuators and the separated shear layer. The measurements were also performed to quantify some of the observations from the oil flow visualization studies and to increase the spanwise measurement region compared to the 2D PIV measurements presented in Section B. With the cameras above the tunnel ceiling, our field of view was obstructed by the wind tunnel frame. Therefore, we shifted the field of view in the $y / s$ direction to be closer to $y / s=0.68$ rather than the centerspan where the 2D PIV measurements were acquired. Because of this shift, the PIV results will not be isolated from the influence of the wing tip vortex. Studies characterizing the interaction between the jet of a fluidic oscillator and a flat plate boundary layer flowfield with and without a pressure gradient have been performed by Ostermann et al. ${ }^{23}$ and Wilson et al. ${ }^{24}$ In the current investigation, we take advantage of the configuration in the BART facility to obtain actuator interaction information recognizing that in addition to the wing tip vortex, the wing flowfield has an adverse pressure gradient and a spanwise flow component in the region where the excitation is introduced. Figure 13(a) shows the wing with the PIV mean contours for $\delta_{f}=40^{\circ}$. Figs. $13(\mathrm{~b})$ and $13(\mathrm{c})$ are included to illustrate the flap coordinate systems and the PIV measurement planes (vertical lines) used for the $\delta_{f}=40^{\circ}$ and $\delta_{f}=60^{\circ}$ configurations, respectively. 


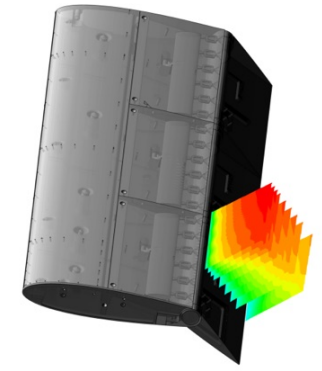

(a) Spanwise PIV measurement region.

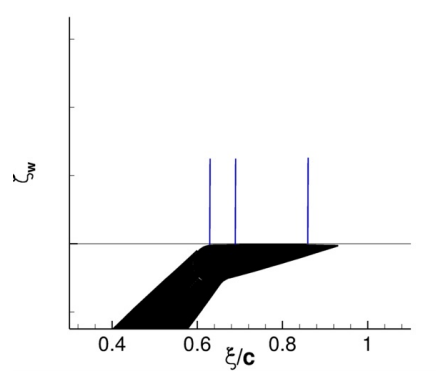

(b) $\delta_{f}=40^{\circ}$ flap coordinate system.

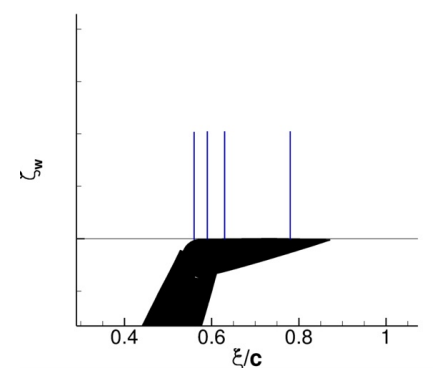

(c) $\delta_{f}=60^{\circ}$ flap coordinate system.

Figure 13. Spanwise PIV measurement region.

\section{A. $\delta_{f}=40^{\circ}$}

Figure 14 presents contours of both velocity and vorticity for the baseline and SWJ controlled flowfields for $\delta_{f}=40^{\circ}$. This flap deflection is being described because it represents a case where AFC is able to reattach the flow to the flap surface. Two amplitude levels are included to show the influence of $C_{\mu}$. Figures 14(a) to $14(\mathrm{c})$ provide the baseline velocity $\left(U_{\xi}\right)$ components in the flap-aligned coordinate system and Figs. 14(g) to 14(i) are the corresponding baseline vorticity $\left(\Omega_{\xi}\right)$ contours. The $\Omega_{\xi}$ contours are provided with velocity vectors overlayed that are computed from the velocity components in the $y$ and $\zeta$ directions Both depict an increase in separated flow on the flap surface as distance from the flap shoulder is increased. SWJ actuation at $C_{\mu}=1.8 \%$ was applied to control separation. The spatially oscillating jets introduce pairs of positive (clockwise on the left) and negative (counter-clockwise on the right) streamwise vortices into the flowfield that promote mixing between the separated shear layer and the low momentum fluid near the flap surface. As mentioned previously, the SWJ actuators are not synchronized so there is a random phase between SWJ actuators. Figures $14(\mathrm{~d})$ to $14(\mathrm{f})$ show $U_{\xi}$ contours for the PIV data acquired at the same streamwise locations as the baseline $\left(C_{\mu}=0 \%\right)$ contours. Near the actuation location (Fig. 14(d)), the output of each actuator is denoted by the concentrations of higher values of $U_{\xi}$ in the contours. The region between SWJ actuators is marked by local pockets of lower speed flow. SWJ AFC is not as effective at $y / s=0.77$ leading to a local region of separated flow that grows with downstream distance. The corresponding mean $\Omega_{\xi}$ contours in Figs. 14(j) to 14(1), show a pair of opposite-signed vortices that represent the crossflow induced by each SWJ actuator. The separated region of the flow at $\xi / c=0.63, y / s=0.77$ results in a pair of vortices at $\xi / c=0.86$ (Fig. 14(1)) that cover a much wider spanwise distance and have a height comparable to the baseline separated shear layer shown in Fig. 14(i). Vorticity contours presented in Figs. 14(m) to 14(o) show that SWJ AFC with $C_{\mu}=2.9 \%$ improves the spanwise uniformity of the streamwise vortices resulting in flow attachment to the trailing edge of the flap surface, in agreement with the $C_{p}$ distributions of Figs. 8(c) and $8(\mathrm{~d})$.

\section{B. $\delta_{f}=60^{\circ}$}

Figure 15 presents the baseline and AFC contours from PIV results obtained when introducing SWJ excitation from the flap shoulder of the model for $\delta_{f}=60^{\circ}$. The baseline velocity (Figs. 15(a) to 15(c)) and vorticity (Figs. $15(\mathrm{~g})$ to $15(\mathrm{i})$ ) contours show the amount of separated flow that is present at $\delta_{f}=60^{\circ}$ prior to control being applied. The shear layers in Figs. 15(g) and 15(h) have braided structures with wavelengths that corresponds to the actuator spacing. The pattern could be due to some localized secondary flows induced by the actuator exit geometry. Similar results were obtained with the actuators sealed with tape thus eliminating actuator cavity resonance as a possible cause. The SWJ locations in Fig. 15(d) coincide with the regions where the local velocity levels exceed the freestream velocity, due to the proximity of this measurement station to the actuator exit. The velocity pattern produced by the SWJ excitation is broken by a velocity deficit at $\xi / c=0.56$ and $y / s=0.71$ (Fig. 15(d)). The separated flow region grows with downstream distance, eventually spanning the majority of the measurement region at $\xi / c=0.78$ (Figs. 15(f) and 15(l)). This flow pattern was also present in the oil flow results of Fig. 10(c). Note that the model trailing edge for this flap deflection is located at $\xi / c=0.87$. 


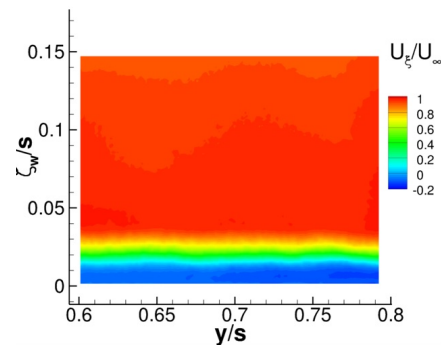

(a) $\xi / c=0.63, C_{\mu}=0 \%$.

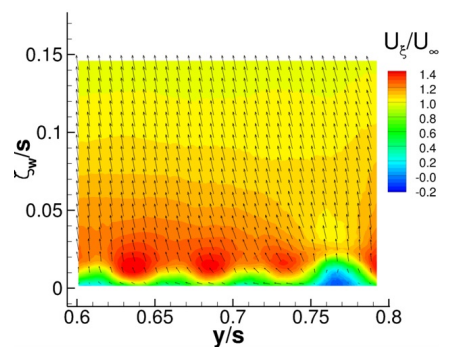

(d) $\xi / c=0.63, C_{\mu}=1.8 \%$.

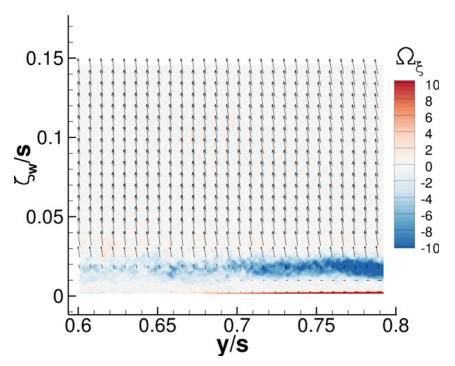

(g) $\xi / c=0.63, C_{\mu}=0 \%$.

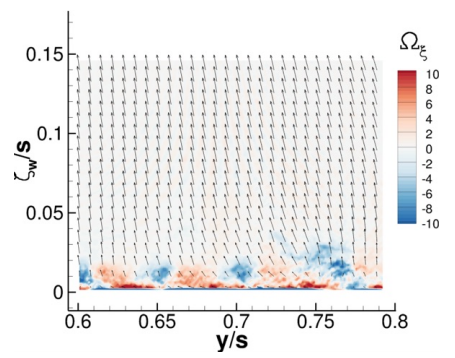

(j) $\xi / c=0.63, C_{\mu}=1.8 \%$.

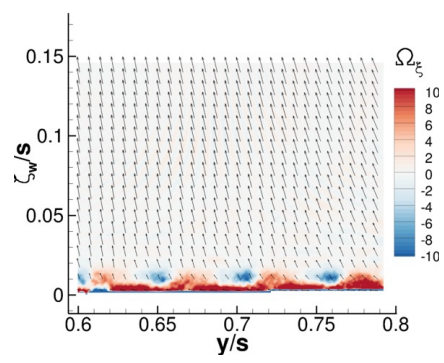

(m) $\xi / c=0.63, C_{\mu}=2.9 \%$.

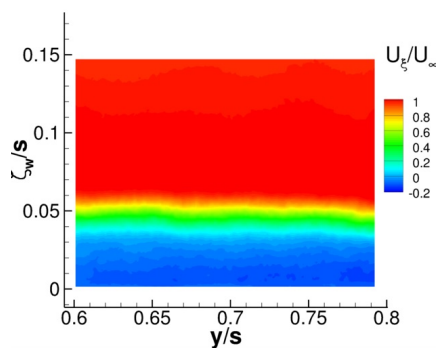

(b) $\xi / c=0.69, C_{\mu}=0 \%$.

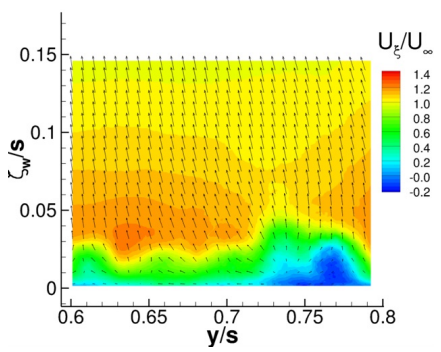

(e) $\xi / c=0.69, C_{\mu}=1.8 \%$.

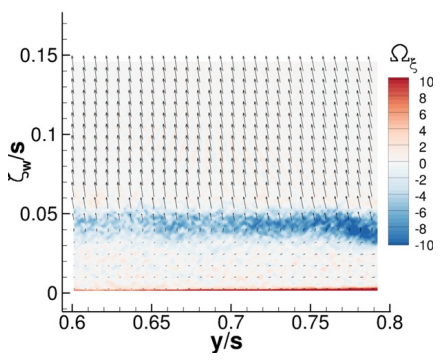

(h) $\xi / c=0.69, C_{\mu}=0 \%$.

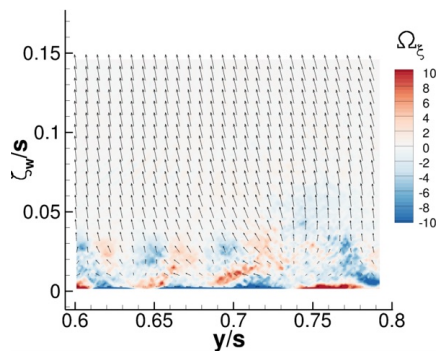

(k) $\xi / c=0.69, C_{\mu}=1.8 \%$.

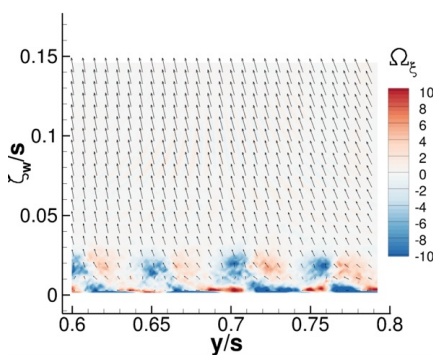

(n) $\xi / c=0.69, C_{\mu}=2.9 \%$.

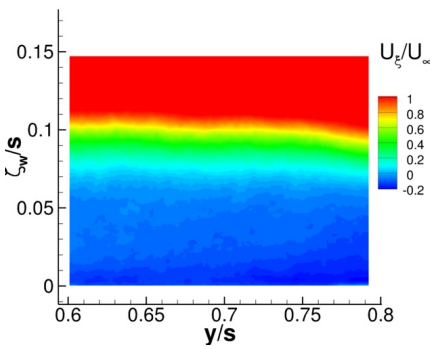

(c) $\xi / c=0.86, C_{\mu}=0 \%$.

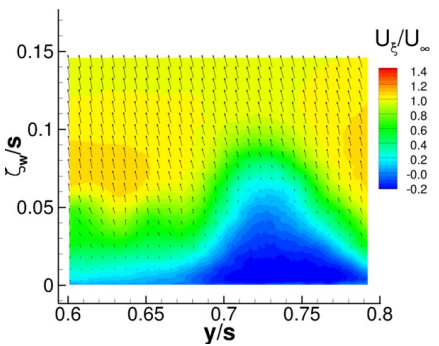

(f) $\xi / c=0.86, C_{\mu}=1.8 \%$.

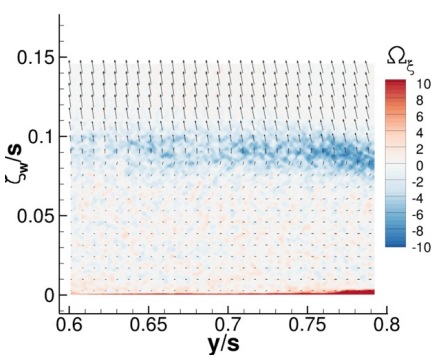

(i) $\xi / c=0.86, C_{\mu}=0 \%$.

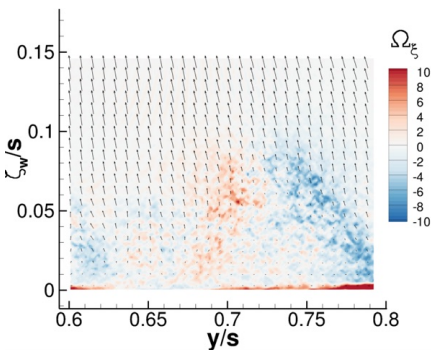

(l) $\xi / c=0.86, C_{\mu}=1.8 \%$.

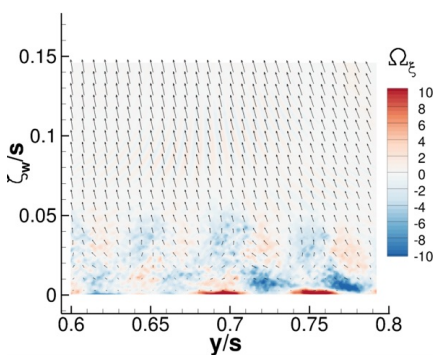

(o) $\xi / c=0.86, C_{\mu}=2.9 \%$.

Figure 14. PIV contours of velocity and vorticity comparing the baseline flowfield to the SWJ controlled flowfield, $\delta_{f}=40^{\circ}, \alpha=8^{\circ}$, and $R e_{c}=500,000$. Every 6th vector in the vertical and horizontal directions displayed for clarity. 


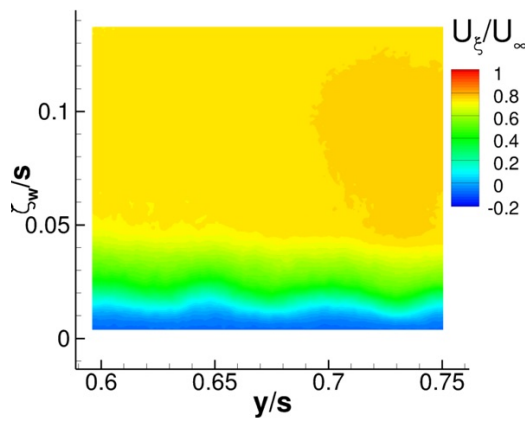

(a) $\xi / c=0.56, C_{\mu}=0 \%$.

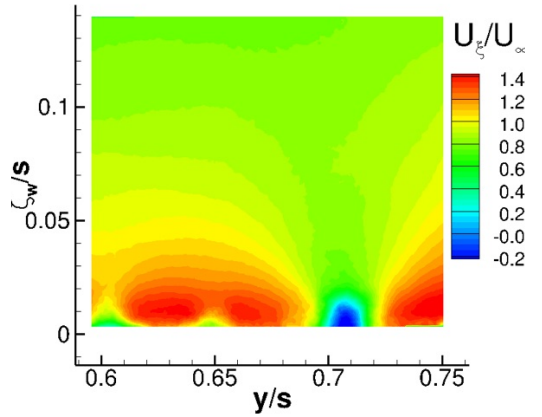

(d) $\xi / c=0.56, C_{\mu}=3.7 \%$

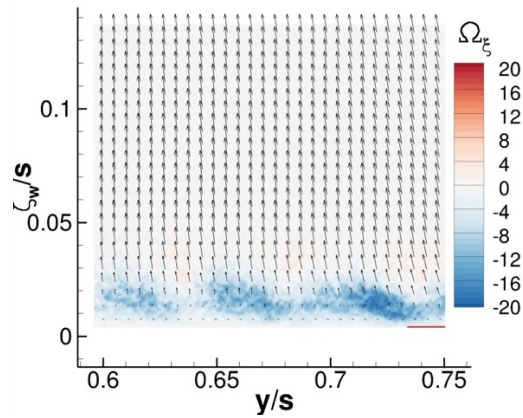

(g) $\xi / c=0.56, C_{\mu}=0 \%$.

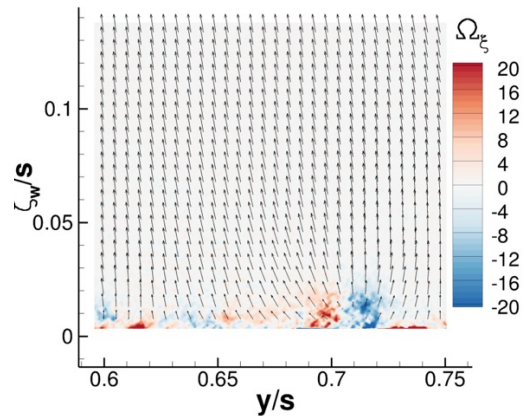

(j) $\xi / c=0.56, C_{\mu}=3.7 \%$.

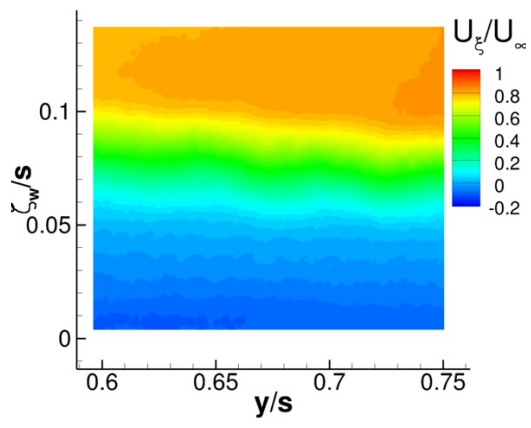

(b) $\xi / c=0.63, C_{\mu}=0 \%$.

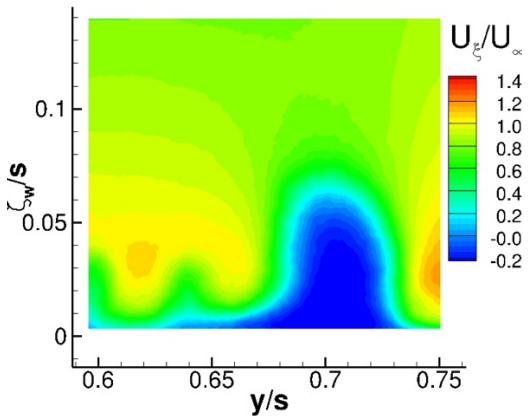

(e) $\xi / c=0.63, C_{\mu}=3.7 \%$.

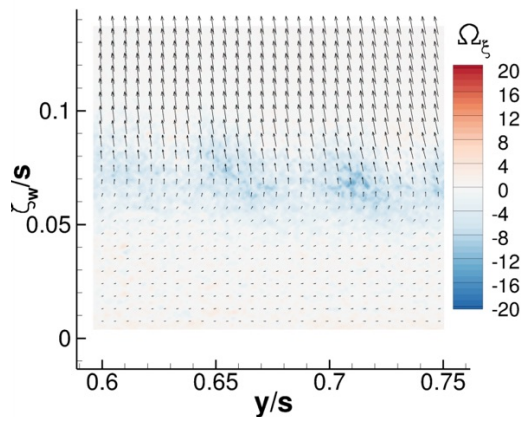

(h) $\xi / c=0.63, C_{\mu}=0 \%$.

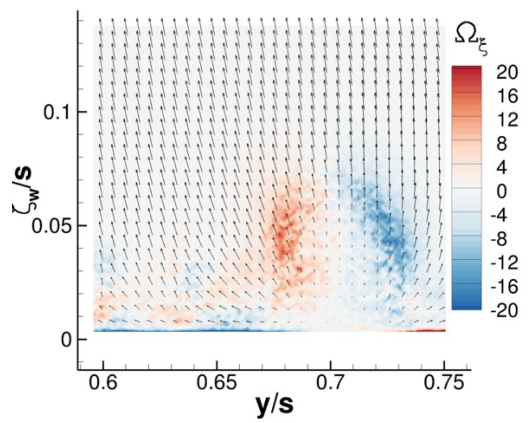

(k) $\xi / c=0.63, C_{\mu}=3.7 \%$.

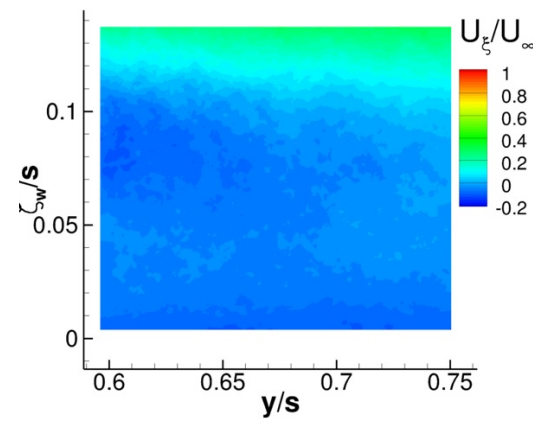

(c) $\xi / c=0.78, C_{\mu}=0 \%$.

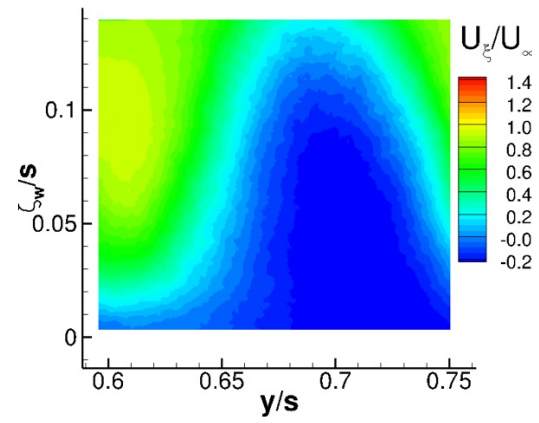

(f) $\xi / c=0.78, C_{\mu}=3.7 \%$.

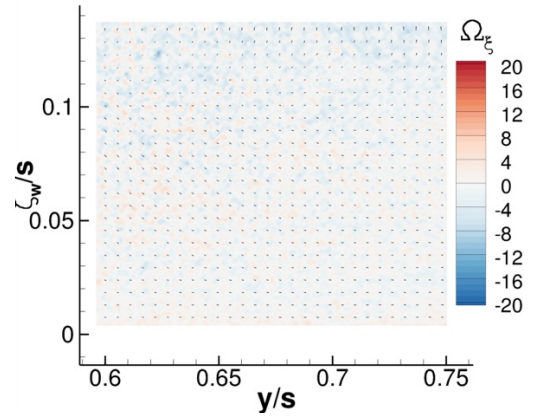

(i) $\xi / c=0.78, C_{\mu}=0 \%$.

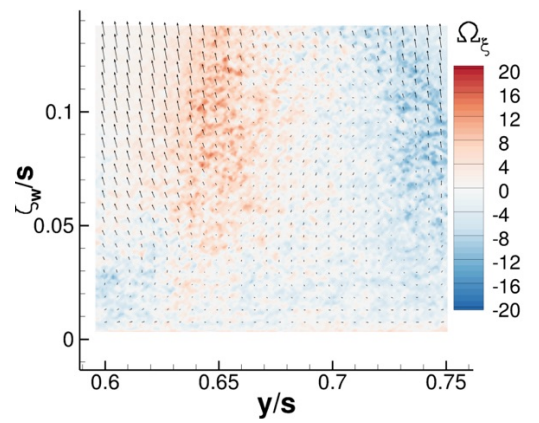

(l) $\xi / c=0.78, C_{\mu}=3.7 \%$.

Figure 15. Velocity and vorticity contours from PIV results comparing the baseline flowfield to the SWJ controlled flowfield, $\delta_{f}=60^{\circ}, \alpha=8^{\circ}$, and $R e_{c}=500,000$. Every 6th vector in the vertical and horizontal directions displayed for clarity. 
Figure 16 presents velocity and vorticity contours acquired at $\xi / c=0.59$ that illustrate the effect of amplitude on the streamwise vortices produced by the SWJ actuators. Velocity $\left(U_{\xi}\right)$ and vorticity $\left(\Omega_{\xi}\right)$ contours (Figs. 16(a) and 16(d)) for the baseline flowfield are also included to show the position of the separated shear layer relative to the AFC flowfield. The contours show that at $C_{\mu}=1.8 \%$ (Fig. 16(e)) the actuators do not create vortices similar to the ones generated at $\delta_{f}=40^{\circ}$ (Figs. 14(k) and 14(n)) when separation was controlled. Rather, the excitation produces discrete regions of attached flow between regions of separated flow characterized by pairs of positive and negative vorticity concentrations similar to the results obtained at $\delta_{f}=40^{\circ}$ with $C_{\mu}=1.8 \%$ (Fig. $14(\mathrm{l})$ ) and at $\delta_{f}=60^{\circ}$ with $C_{\mu}=3.7 \%$ (Fig. $15(\mathrm{l})$ ). As $C_{\mu}$ is increased, the $U_{\xi}$ contours of Figs. 16(b) and 16(f) show that the amount of separated flow is reduced and two small regions of separated flow near $y / s=0.6$ and 0.7 remain at $C_{\mu}=3.6 \%$.

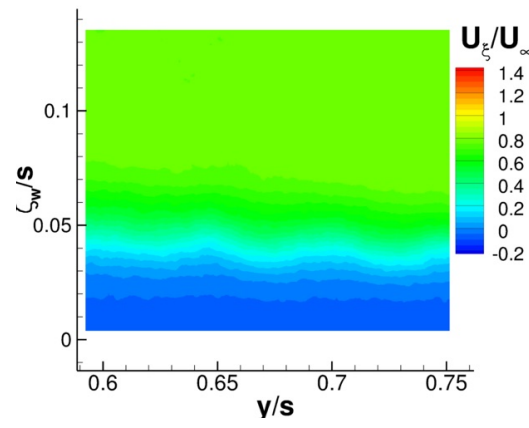

(a) $C_{\mu}=0 \%$.

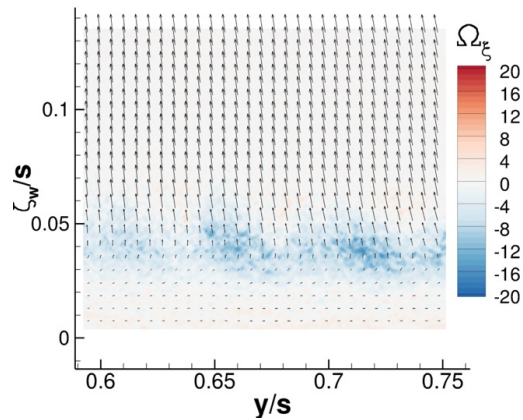

(d) $C_{\mu}=0 \%$.

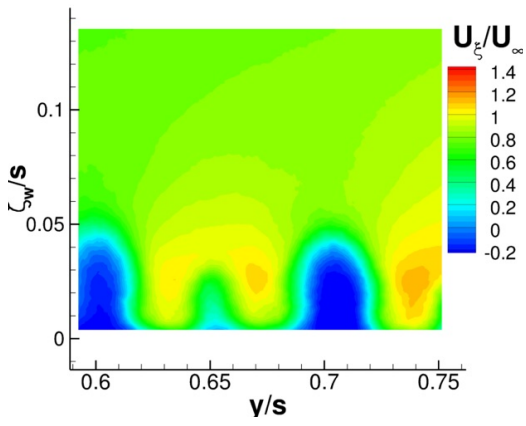

(b) $C_{\mu}=1.8 \%$.

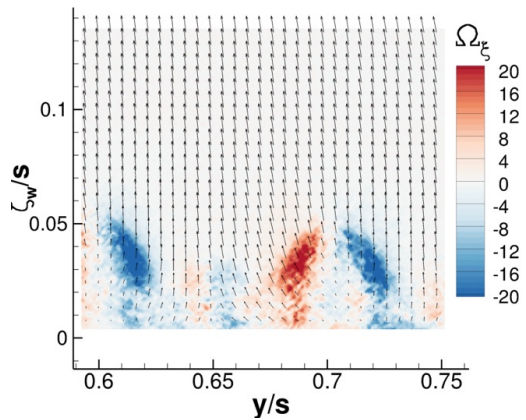

(e) $C_{\mu}=1.8 \%$.

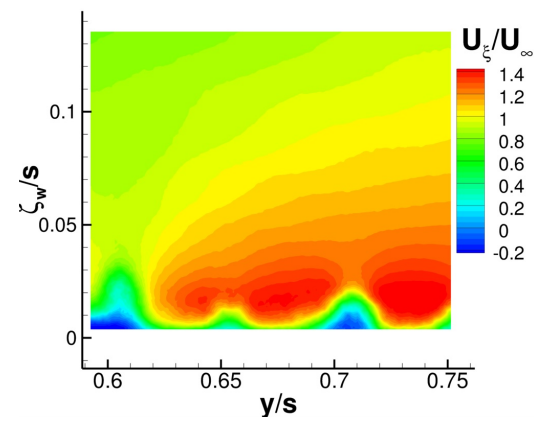

(c) $C_{\mu}=3.6 \%$.

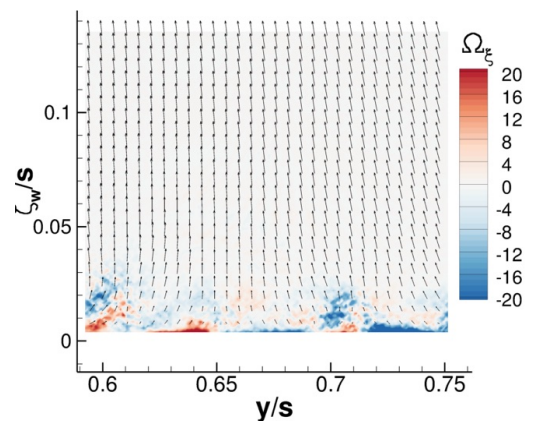

(f) $C_{\mu}=3.6 \%$.

Figure 16. Velocity and vorticity contours from PIV measurements at $\xi / c=0.59$ using SWJ actuators and showing the effect of varying $C_{\mu}, \delta_{f}=60^{\circ}, \alpha=8^{\circ}$, and $R e_{c}=500,000$. Every 6th vector in the vertical and horizontal directions displayed for clarity.

Figure 17 presents velocity and vorticity contours acquired at $\xi / c=0.59$ obtained from PIV measurements with CD AFC applied. The baseline $U_{\xi}$ and $\Omega_{\xi}$ are included for reference. Figure 17(e) shows that at each actuator location, concentrations of positive and negative vorticity are introduced into the flowfield with the $C_{\mu}=3.3 \%$ excitation. The excitation reduces locally the amount of separated flow at the jet exit as depicted in the $U_{\xi}$ contours of Fig. 17(b). The small region of influence is due in part to the jet spreading angle being small ( $P R=1.4$ ). Positive vorticity is produced on the right side of the actuator and negative vorticity is produced on the left side (Fig $17(\mathrm{e})$ ). These signs are opposite the signs of the vorticity produced by the SWJ actuators (Figs. 14(k) and 14(n)). The small negative vorticity concentration on the right side of the vortex pattern, produced by each actuator, is not present at low $C_{\mu}$ values. Once present, however, it grows in strength and size as $C_{\mu}$ increases, until the flow is attached to the flap surface. The $C_{\mu}=6.6 \%$ excitation attaches the shear layer to the flap surface at this location over most of the measurement region. The attached flowfield of Fig. 17(f) has positive-signed vortices aligned in the spanwise direction with the CD actuator exits. Although not shown, CD excitation is not as effective at controlling separation downstream of this location due to the vorticity, introduced into the flowfield by the actuation, being too far from the flap surface. 


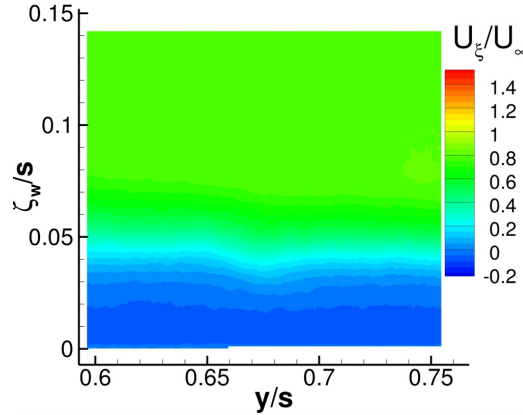

(a) Baseline.

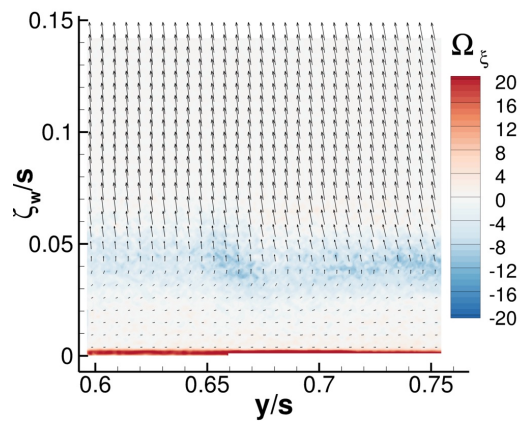

(d) Baseline.

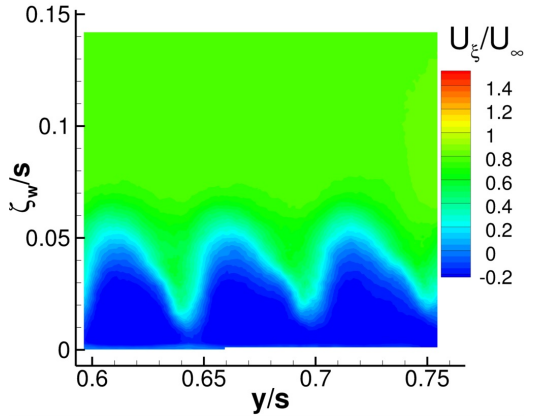

(b) $C_{\mu}=3.3 \%$.

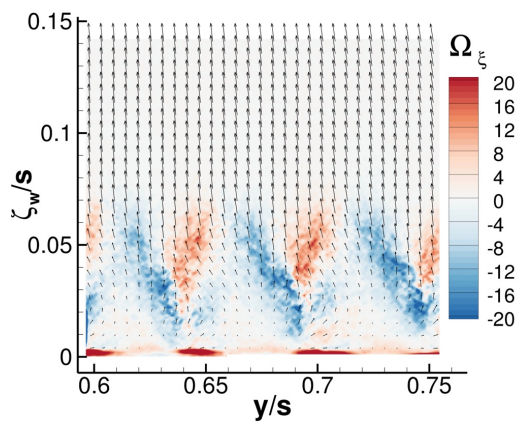

(e) $C_{\mu}=3.3 \%$.

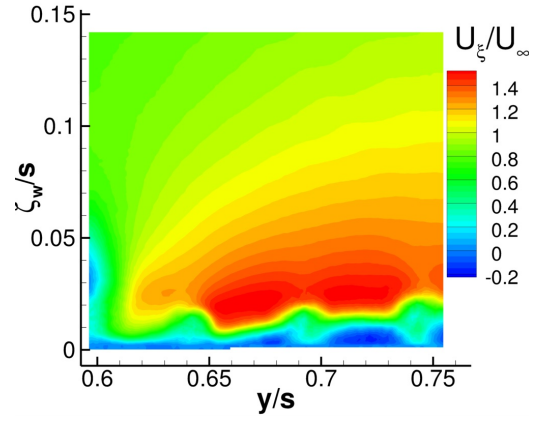

(c) $C_{\mu}=6.6 \%$.

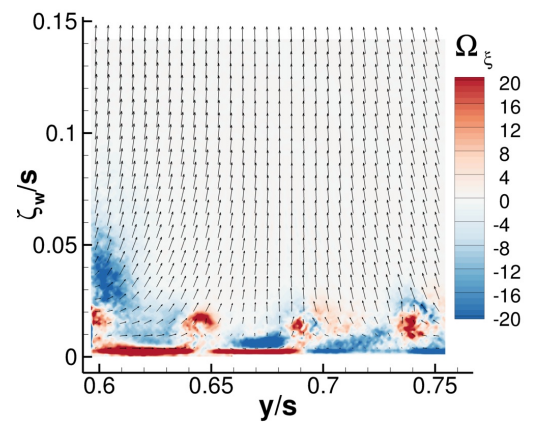

(f) $C_{\mu}=6.6 \%$.

Figure 17. Velocity and vorticity contours from PIV measurements at $\xi / c=0.59$ using the CD actuators showing the effect of varying $C_{\mu}, \delta_{f}=60^{\circ}, \alpha=8^{\circ}$, and $R e_{c}=500,000$. Every 6th vector in the vertical and horizontal directions displayed for clarity. 


\section{Summary}

Flow physics experiments were performed on a semispan, unswept NACA 0015 with a hinged, $30 \%$ chord, trailing edge flap to study the interaction between active flow control (AFC) actuation and the separated shear layer resulting from deflecting the flap. Previous experiments had been performed on the model geometry but were limited to a $\delta_{f}=40^{\circ}$. The primary focus of the current experiments was to assess the effectiveness of AFC to control separation on the $\delta_{f}=60^{\circ}$ configuration, in support of a large-scale high-lift AFC experiment. Steady jet (CD) and sweeping jet (SWJ) actuators with a fixed spacing of $3.3 \mathrm{~cm}$ were used for the study. Two jet cores, angled with respect to the actuator center, were present in the SWJ actuator mean total pressure contours acquired during the benchtop characterization studies. In comparison, the CD actuator generated a steady straight jet with a spreading angle that increased when sonic conditions were present in the nozzle. The maximum measured oscillation frequency of the SWJ actuator was 1500 $\mathrm{Hz}$, a value more than an order of magnitude larger than the natural instabilities in the flowfield. SWJ AFC excitation, introduced from the flap shoulder was effective at flow separation control at lower flap deflections of $20^{\circ}$ and $40^{\circ}$. However, at the higher flap deflection of $60^{\circ}$, flow attachment over the entire flap surface could not be obtained with the actuation methods investigated using a $P R \leq 2.7$. Nevetheless, both actuators produced an increment in lift, with the sweeping jet actuators requiring less input pressure and mass flow. Oil flow images showed that this was due, in part, to the fact that the SWJ actuators cover a larger extent of the flap span and produce streamwise vortices that, along with the momentum introduced by the actuators, make them effective at separation control. Two-dimensional Particle Image Velocimetry results showed that excitation from the flap shoulder was not optimal for the $60^{\circ}$ flap deflection case. Consequently the momentum and the distance between the streamwise vortices introduced by the excitation and flap surface increased with downstream distance. Additionally, the steady discrete CD excitation configuration of this study had a detrimental impact on lift at the highest momentum input levels investigated. The $C_{\mu}$ levels used for this case and most of the $\delta_{f}=60^{\circ}$ cases exceeded, by far, the levels that are commonly used for active separation control. This approach was taken because we were interested in understanding the flow attachment process and the actuation requirements for the large flap deflection and thus adverse pressure gradients of this study. Stereoscopic PIV results were used to determine, at least in a mean sense, how the excitation interacted with the flap flowfield. The $\delta_{f}=40^{\circ}$ case was used as a reference because it represented a case where SWJ AFC was capable of attaching the flow to the flap surface. With effective separation control, a pair of vortices located on either side of the jet centerline was present in the flowfield. When separation existed on the flap, as was the case for the $\delta_{f}=60^{\circ}$, the flap flowfield was three-dimensional (3D), as seen in both the oil flow and the PIV results. Near the flap shoulder, where both actuation methods effectively attached the flow to the flap surface, positive-signed vortices, aligned with the actuator centerlines, were present in the flowfield controlled by the CD actuators. The results of this study suggest that additional research should include studies with the excitation closer to tangential to the flap surface and AFC studies using multiple rows of actuators to distribute the actuation momentum along the flap surface, with the goal of reducing the overall momentum requirements.

\section{Acknowledgments}

The authors would like to thank the Advanced Air Transport Technology Project of the NASA Advanced Air Vehicles Program for funding the research and the following individuals for their support; Catherine McGinley, Luther Jenkins, Judi Hannon, Norman Schaeffler, Christal Kellam, Charlie Debro, and Donald Day. Thanks are also extended to Mark Fletcher and Chung-sheng Yao for help with the PIV acquisition and analysis. The authors also thank model designer Sandy Webb for his work on the SWJ actuator arrays. Thanks are extended to Wayne Geouge, Tom Hall, Kevin McLain, Robert Andrews, and Gary Wainwright for help with various aspects of the model fabrication and instrumentation process. Finally, thanks are extended to Louis Edelman for the actuator characterization software.

\section{References}

\footnotetext{
${ }^{1}$ McClean, J. D., Crouch, J. D., Stoner, R. C., Sakurai, S., Feifel, G. E., Feifel, W. M., and Rush, H. M., "Study of the Application of Separation Control by Unsteady Excitation to Civil Transport Aircraft," NASA/CR 1999-209338, 1999.

${ }^{2}$ Greenblatt, D., "Dual Location Separation Control on a Semispan Wing," AIAA Journal, Vol. 45, No. 8, 2007, pp. 1848-
} 
1860.

${ }^{3}$ Melton, L. P. and Koklu, M., "Active Flow Control Using Sweeping Jet Actuators on a Semi-Span Wing Model," AIAA Paper 2016-1872, Jan. 2016.

${ }^{4}$ Melton, L. P., Koklu, M., Andino, M., Lin, J. C., and Edelman, L. M., "Sweeping Jet Optimization Studies," AIAA Paper 2016-4233, June 2016.

${ }^{5}$ Andino, M. Y., Lin, J. C., Washburn, A. E., Whalen, E. A., Graff, E. C., and Wygnanski, I. J., "Flow Separation Control on a Full-Scale Vertical Tail Model using Sweeping Jet Actuators," AIAA Paper 2015-0785, Jan. 2015.

${ }^{6}$ Whalen, E. A., Lacy, D. S., Lin, J. C., Andino, M. Y., Washburn, A. E., Graff, E. C., and Wygnanski, I. J., "Performance Enhancement of a Full-Scale Vertical Tail Model Equipped with Active Flow Control," AIAA Paper 2015-0784, Jan. 2015.

${ }^{7}$ Lin, J. C., Andino, M. Y., Alexander, M. G., Whalen, E. A., Spoor, M. A., Tran, J. T., and Wygnanski, I. J., "An Overview of Active Flow Control Enhanced Vertical Tail Technology Development," AIAA Paper 2016-0056, Jan. 2016.

${ }^{8}$ Nagib, H., Kiedaisch, J., Reinhard, P., and Demanett, B., "Active Flow Control for High Lift Airfoils: Separation versus Circulation Control," AIAA Paper 2007-1119, Jan. 2007.

${ }^{9}$ Melton, L. P., Yao, C., and Seifert, A., "Active Control of Separation from the Flap of a Supercritical Airfoil," AIAA Journal, Vol. 44, No. 1, 2006, pp. 34-41, previously AIAA paper 2003-4005.

${ }^{10}$ Hartwich, P. M., Shmilovich, A., Lacy, D. S., Dickey, E. D., Sclafani, A. J., Sundaram, P., and Yadlin, Y., "Refined AFC-Enabled High-Lift System Integration Study," NASA/CR 2016-219170, 2016.

${ }^{11}$ Woszidlo, R. and Wygnanski, I., "Parameters Governing Separation Control with Sweeping Jet Actuators," AIAA Paper 2011-3172, June 2011.

${ }^{12}$ Gregory, J. W. and Tomac, M., "A Review of Fluidic Oscillator Development and Application for Control," AIAA Paper 2013-2474, June 2013.

${ }^{13}$ Lin, J. C., Melton, L. P., Viken, S., Andino, M. Y., Koklu, M., Hannon, J., and Vatsa, V. N., "High Lift Common Research Model for Wind Tunnel Testing: An Active Flow Control Perspective," AIAA Paper 2017-0319, Jan. 2017.

${ }^{14}$ Greenblatt, D. and Washburn, A., "Influence of Finite Span and Sweep on Active Flow Control Efficacy," AIAA Journal, Vol. 46, No. 7, 2008, pp. 1675-1694.

${ }^{15}$ Greenblatt, D., "Management of Vortices Trailing Flapped Wings via Separation Control," AIAA Paper 2005-0061, Jan. 2005.

${ }^{16}$ Jones, G. S., Milholen, W. E., Chan, D. T., and Goodliff, S. L., "A Sweeping Jet Application on a High Reynolds Number Semi-Span Supercritical Wing Configuration," AIAA Paper 2017-3044, June 2017.

${ }^{17}$ Wernet, M. P., "Fuzzy Logic Enhanced Digital PIV Processing Software," 18th International Congress on Instrumentation for use in Aerospace Simulation Facilities 2010-4247, June 1999.

${ }^{18}$ Scarano, F. and Riethmuller, M., "Advances in Iterative Multigrid PIV Image Processing," Experiments in Fluids Supplemental, Vol. 29, 2000, pp. S51-S60.

${ }^{19}$ Koklu, M., "Effect of a Coanda Extension on the Performance of a Sweeping-Jet Actuator," AIAA Journal, Vol. 54, No. 3, March 2016, pp. 1131-1134.

${ }^{20}$ Wassermann, F., Hecker, D., Jung, B., Markl, M., Seifert, A., and Grundmann, S., "Phase-locked 3D3C-MRV measurements in a bi-stable fluidic oscillator," Experiments in Fluids, Vol. 54, No. 3, March 2013, pp. 1107-15.

${ }^{21}$ Ostermann, F., Woszidlo, R., Nayeri, C. N., and Paschereit, C. O., "Phase-Averaging Methods for the Natural Flowfield of a Fluidic Oscillator," AIAA Journal, Vol. 53, No. 8, Aug. 2015, pp. 2359-2368.

${ }^{22}$ Vatsa, V., Koklu, M., and Wygnanski, I., "Numerical Simulation of Fluidic Actuators for Flow Control Applications," AIAA Paper 2012-3239, June 2012.

${ }^{23}$ Ostermann, F., Woszidlo, R., Nayeri, C., and Paschereit, C. O., "The Time-Resolved Flow Field of a Jet Emitted by a Fluidic Oscillator into a Crossflow," AIAA Paper 2016-0345, Jan. 2016.

${ }^{24}$ Wilson, J., Schatzman, D. M., Seifert, A., Arad, E., Marom, L., Shay, N., and Palei, V., "Characterization of SaOB Actuators Interacting With Flat Plate Boundary Layers," AIAA Paper 2017-1691, Jan. 2017. 